\title{
CONSERVATION LAWS OF FREE BOUNDARY PROBLEMS AND THE CLASSIFICATION OF CONSERVATION LAWS FOR WATER WAVES
}

\author{
BY \\ PETER J. OLVER ${ }^{1}$
}

\begin{abstract}
The two-dimensional free boundary problem for incompressible irrotational water waves without surface tension is proved to have exactly eight nontrivial conservation laws. Included is a discussion of what constitutes a conservation law for a general free boundary problem, and a characterization of conservation laws for two-dimensional free boundary problems involving a harmonic potential proved using elementary methods from complex analysis.
\end{abstract}

Introduction. The main purpose of this paper is to prove that the free boundary problem describing the motion of gravity waves over a two-dimensional irrotational, incompressible ideal fluid in the absence of surface tension ("water waves") has exactly eight independent conservation laws. Extensions to three-dimensional waves, with or without surface tension are indicated, but not explicitly proven. This result carries a number of implications for the interpretation of the qualitative and quantitative properties of real water waves by soliton models such as the KortewegdeVries equation, which we discuss at length in $\$ 2$.

The proof of such a result must incorporate a precise definition of the concept of a conservation law for a free boundary problem, which, to my knowledge, has not appeared in the literature to date. $\$ 3$ elaborates on the physical and mathematical motivations for the definition proposed here, which is more general than what one might, by analogy with the corresponding concept for systems of partial differential equations, be tempted to use. The present definition of a conservation law is formulated so as to be applicable to a wide class of free boundary problems.

A second result of more general applicability is an interesting characterization of conservation laws for two-dimensional free boundary problems in which the field variables consist of a single harmonic potential. In essence, the time derivative of the conserved density must equal the sum of a divergence and an analytic contribution, the latter being the unusual feature of this result; see $\$ 5$.

I would like to thank T. Brooke Benjamin for the vital encouragement needed to complete this work.

Received by the editors November 20, 1981 and, in revised form, May 3, 1982.

1980 Mathematics Subject Classification. Primary 35R35, 35Q20, 76B15.

Key words and phrases. Free boundary problem, conservation law, water waves, completely integrable, harmonic function.

${ }^{1}$ Research supported in part by National Science Foundation Grant NSF MCS 81-00786. 
1. The water wave problem. Consider the free boundary problem describing the motion of surface waves over a two-dimensional incompressible, irrotational fluid flow under the influence of gravity in the absence of surface tension. The fluid moves in a fixed container determined by a domain $\Omega_{0} \subset \mathbf{R}^{2}$ with boundary $\Gamma_{0}$. The domain $\Omega_{0}$ can have unbounded components, so, for example, $\Omega_{0}=\mathbf{R}^{2}$ for waves on an ocean of infinite depth, or $\Omega_{0}=\{(x, y): y \geqslant-h\}$ for the case of waves over a horizontal bottom. The free surface $S$ is assumed to be described by a single valued function $y=\eta(x, t)$, so that at any given time the fluid occupies the domain $\Omega=\left\{(x, y) \in \Omega_{0}: y \leqslant \eta(x, t)\right\}$. (This assumption is in fact inessential, cf. $\S 6$, so generalizations to parametrically described surfaces are straightforward.) Because the motion remains irrotational by Kelvin's theorem, the Eulerian velocity field $\mathbf{u}(x, y, t)$ possesses a potential function $\varphi(x, y, t)$ defined in $\Omega$ with $\nabla \varphi=\mathbf{u}$. Incompressibility requires $\varphi$ to be harmonic, so Laplace's equation

$$
\Delta \varphi=0 \text { in } \Omega
$$

holds throughout the domain. Boundary conditions on any portion $\Gamma$ of the fixed boundary $\Gamma_{0}$ bounding $\Omega$ are presumed, usually consisting of the no seepage condition

$$
\partial \varphi / \partial n=0 \quad \text { on } \Gamma
$$

together with appropriate decay conditions for $\varphi$ on any unbounded portion of $\Omega$. The exact nature of these fixed boundary conditions is not essential for our results. Finally the free surface $S$ is determined by two nonlinear equations: a dynamical condition

$$
\varphi_{t}+\frac{1}{2}|\nabla \varphi|^{2}+g \eta=0 \text { on } S
$$

(subscripts denoting derivatives) reflecting the vanishing of the pressure at the free surface ( $g>0$ being the gravitational constant), and a kinematic condition

$$
\eta_{\mathbf{t}}=\varphi_{y}-\eta_{\mathbf{x}} \varphi_{x} \quad \text { on } S
$$

prescribing the motion of $S$ to be in the direction of the normal component of velocity. (See $\$ 3$ for an explanation of the use of boldface subscripts for derivatives on the surface.) Equations (1.1)-(1.4) constitute the water wave problem; further details on their derivation may be found in texts on hydrodynamics, e.g. [12, 25].

The concept of a conservation law for the free boundary problem (1.1)-(1.4) will be precisely defined in $\$ 3$, but, in essence, a conservation law is given by an integral $\int_{S} T d x$ over the free surface, $T$ being the conserved density, whose time variation depends only on the behavior of the solution on the fixed boundary $\Gamma$ (or the asymptotic behavior at large distances in the case of unbounded domains). Benjamin and Olver [4] combined a Hamiltonian formulation of the water wave problem due to Zakharov [30] with symmetry group theory to find eight independent nontrivial 
conservation laws. The eight conserved densities for these laws are

$$
\begin{aligned}
& T_{1}=\eta_{\mathrm{x}} \varphi \quad \text { (horizontal momentum), } \\
& T_{2}=\frac{1}{2} \varphi\left(\varphi_{y}-\eta_{\mathrm{x}} \varphi_{x}\right)+\frac{1}{2} g \eta^{2} \quad \text { (energy), } \\
& T_{3}=\eta \quad \text { (mass), } \\
& T_{4}=\varphi+t T_{3} \quad \text { (vertical momentum), } \\
& T_{5}=x \eta-t T_{1} \quad \text { (center of mass), } \\
& T_{6}=\frac{1}{2} \eta^{2}-t T_{4}+\frac{1}{2} g t^{2} T_{3} \quad \text { (potential energy), } \\
& T_{7}=\left(x \eta_{\mathrm{x}}-\eta\right) \varphi+t\left(4 T_{2}-7 g T_{6}\right)-\frac{7}{2} g t^{2} T_{4}+\frac{7}{6} g^{2} t^{3} T_{3} \quad \text { (virial), } \\
& T_{8}=\left(x+\eta \eta_{\mathrm{x}}\right) \varphi+g t T_{5}-\frac{1}{2} g t^{2} T_{1} \quad \text { (angular momentum). }
\end{aligned}
$$

Of these, the first three are well known. Benjamin and Mahony [3] proved the conservation of $T_{5}$ for a wide class of fluid mechanical problems. Longuet-Higgins introduced $T_{6}$ in his study of pressure variations at large depths [13] and, later, $T_{8}$ in a study of breaking waves [14]. The density $T_{7}$, which is related to a "virial theorem" for water waves, is new. A more thorough discussion of the physical interpretations and applications of these laws is done in [4], to which we refer the reader for details.

In this paper it will be shown that the water wave problem has no further nontrivial conservation laws. In other words, any other conserved density is equivalent (in a sense to be made precise) to a linear combination of $T_{1}, \ldots, T_{8}$. The precise statement of this result, which depends on our general definition of conservation law, can be found in Theorem 7.1.

The technique of proof of this result is not very difficult conceptually, but involves a lot of hard computational manipulations. The main tool is the characterization theorem of conserved densities of harmonic free boundary problems proved in $\$ 5$. Detailed analysis of the dependence of a conserved density on the highest order derivatives of $\varphi$ and $\eta$ occurring shows that this dependence must be in some sense trivial. This reduces the density to one depending on lower order derivatives of $\varphi$ and $\eta$, and the whole process is repeated. Eventually, the density is reduced to one depending solely on first order derivatives. A final tedious computation shows that it then must be equivalent to a linear combination of the eight known densities. The proof is then complete.

It is somewhat surprising, given the difficulty of proving such a result for systems of partial differential equations, that a complicated free boundary problem such as the water wave problem should be vulnerable to a direct assault using such primitive weapons. The ameliorating factor appears to be the inclusion of complex-analytic terms in the characterization Theorem 5.1, which initially seem to hopelessly complicate the situation. In essence, all computations reduce to the proposition that the derivative of an analytic function is again analytic. This is extremely fortunate, since sophisticated techniques for answering questions about conservation laws of differential equations are not yet available, so brute force calculations are the only resort. (The few general techniques introduced in [18] do not appear to be of any use 
here.) The method of proof clearly extends to a large variety of harmonic free boundary problems, but in the absence of interesting examples I have not pursued this direction in any great detail.

For two-dimensional waves with surface tension there are seven conservation laws. The same techniques will undoubtedly prove that these are the only nontrivial conservation laws, but I have not yet carried through the details. For three-dimensional water waves, the analytic function techniques vital to the proof are no longer available. However, any conserved density in three dimensions must project to a conserved density for a corresponding two-dimensional problem on any two-dimensional surface. The two-dimensional theorem can then be applied. However it must be shown that for each nontrivial three-dimensional density, there is a two-dimensional problem such that the projection of this density remains nontrivial. Space limitations preclude the resolution of this problem here, but this technique should certainly prove that the thirteen conservation laws (twelve if surface tension is included) found by Benjamin and Olver are the only conservation laws for the three-dimensional problem.

2. Implications for soliton theories. While the equations governing the motion of water waves are easy to derive from physical principles, the resulting nonlinear free boundary problem is of such complexity as to have resisted all attempts at solution or even proof of the existence of solutions. (See [17, 21, 22, 24, 29] for some recent results on the latter problem.) To gain insight into the behavior of water waves, one is forced to replace the full equations by a simpler model system. A model for the unidirectional propagation of long waves over shallow water, incorporating both nonlinear and dispersive effects, was proposed by Korteweg and deVries [10] in 1895. This Korteweg-deVries equation was the first of a number of physically relevent model nonlinear wave equations shown to be integrable by the methods of inverse scattering, cf. [11], a discovery which has sparked a revolution in interest and understanding of nonlinear wave interactions. These "soliton" equations share a number of remarkable properties, including clean interaction of solitary wave (soliton) solutions, an infinite number of conservation laws, Bäcklund transformations, etc., and can be viewed as the infinite-dimensional analogues of the classical completely integrable Hamiltonian systems $[9,16]$. It is significant that all of the above properties always appear together in any system, although at present no general proof of this commonly accepted principle exists. If, however, one accepts its validity, then an equation with soliton solutions must have an infinite number of conservation laws. Support for this point of view also comes from the BBM equation [2], an alternative model to the Korteweg-deVries equation, which has been shown [18] to have only three conservation laws. Numerical work of Bona, Pritchard and Scott [6] then showed that its solitary wave solutions do not interact cleanly-a small, but detectable, dispersive tail appears subsequent to any interaction.

The proof here that the full water wave problem has only eight conservation laws therefore indicates that solitary water waves, whose existence was established in $[1,8]$, are not solitons, but probably have qualitative behavior more closely modelled by the BBM equation. Experimental support for this conjecture is no doubt beyond 
the capabilities of even the most conscientious experimenter, so we must await (with great interest) the implementation of a numerical scheme of sufficient accuracy to solve the interaction problem for solitary water waves. ${ }^{2}$

The situation here might well be compared with the history of finite-dimensional Hamiltonian systems, where complete integrability is characterized by a sufficient number of first integrals. After a period of great enthusiasm and interest, much of the physical motivation for studying completely integrable systems was dealt a fatal blow when Bruns and Poincare proved that the $n$-body problem possessed no integrals beyond those of mass, energy and linear and angular momenta and, hence, for $n \geqslant 3$, could not be completely integrable. (See Whittaker [28] for a complete discussion.) These results and the present investigation highlight a seemingly fundamental principle - that the full equations governing nondissipative physical systems are almost never integrable, whereas many approximate models for the systems are. Preliminary evidence on the Euler equations of inviscid fluid flow [20], where the long wave approximation of Benney [5] is integrable [15], lends further support to this principle.

In all cases, the approximation procedure, either linear or nonlinear, introduces unnatural symmetries in the resulting model equations. In the absence of any rigorous pertubation scheme, a cautious attitude towards the indiscriminate application of results for the model equations to the physical system must necessarily be adopted. (For the Korteweg-deVries equation, a rigorous justification of the model, including a result on what, if anything, its infinity of conservation laws imply for the original system, is an outstanding problem.) On the other hand, the appearance of integrable equations as natural approximations to many physical systems indicates that these systems may in some sense be "nearly integrable", so that, for instance, the interaction of solitary wave solutions is, as in the BBM equation, almost perfect. To place this rather vague idea on any firm theoretical basis is undoubtedly extremely difficult, so true understanding of the physical systems lies far in the future.

3. What is a conservation law? For a physical system governed by a system of partial differential equations, the mathematical formulation of the physically important notion of a conservation law is standard. If $x \in \mathbf{R}^{p}, t \in \mathbf{R}$ are the spatial and temporal variables and $u \in \mathbf{R}^{q}$ the field variables of the system, a conservation law is given by an equation of the form

$$
D_{t} T+\operatorname{Div} \mathbf{X}=0
$$

to be satisfied for all solutions $u=f(x, t)$ of the physical system. Here $T$, the conserved density, and $\mathbf{X}=\left(X_{1}, \ldots, X_{p}\right)$, the associated flux, are, in general, functions of $x, t, u$ and the various partial derivatives of $u$. The symbol $D_{t}$ denotes total derivative with respect to $t$ (treating $u$ as a function of $x, t$ ); similarly Div $\mathbf{X}=D_{x_{1}} X_{1}$ $+\cdots+D_{x_{p}} X_{p}$ is the total divergence with respect to the spatial variables $x=$ $\left(x_{1}, \ldots, x_{p}\right)$.

\footnotetext{
${ }^{2}$ Recent work of Mirie and Su, J. Fluid Mech. 115 (1982), 475-492, supports this conclusion.
} 
The reason that an equation of the form (3.1) is a conservation law is that if $u$ is any solution of the physical system decaying sufficiently rapidly for large $|x|$, the quantity $Q=\int_{\mathbf{R}^{p}} T(x, t, u) d x$ is, by the divergence theorem, conserved, i.e. independent of $t$. More generally, considering the solution only over a bounded region $\Omega \subset \mathbf{R}^{p}$ with smooth boundary $\partial \Omega$, the corresponding integral $Q_{\Omega}=\int_{\Omega} T d x$ satisfies

$$
d Q_{\Omega} / d t=\int_{\partial \Omega} \mathbf{X} \cdot d \mathbf{S} .
$$

In other words, the rate of change of the total density $Q_{\Omega}$ over $\Omega$ depends only on the behavior of the solution on the boundary $\partial \Omega$ and in fact equals the total flux over the boundary. Clearly this reflects the general form of a conservation law for a nondissipative physical phenomenon.

For a given system, it is of great interest to find and classify all its conservation laws. If $T$ is itself a divergence, $T=\operatorname{Div} \mathbf{Y}$, for all solutions of the equations, then an equation of form (3.1) clearly holds. Such densities are called trivial and we need only consider the classification of nontrivial conservation laws. Applications of conservation laws to problems of existence and decay of solutions are numerous; for instance see Strauss [26] for some recent applications to nonlinear wave equations.

Many important systems in hydrodynamics, such as the equations for water waves, are governed, not by a system of partial differential equations, but by a free boundary problem. For the general type of problem to be considered here, the spatial variables include a distinguished vertical direction $y \in \mathbf{R}$ as well as one or more horizontal coordinates $x \in \mathbf{R}^{p}$. The free ( $p$-dimensional) surface $S$ is described by a single valued function $y=\eta(x, t)$ whose determination is part of the problem. At time $t$ the fluid will occupy a domain $\Omega_{t}=\Omega$ bounded above by the position of the free surface $S$ at the given time and below by any fixed boundaries in the problem, for example a horizontal bottom. The constitutive relations and properties of the fluid will determine a system of partial differential equations

$$
\Delta(x, y, t, u)=0
$$

to be satisfied throughout the domain $\Omega$. In addition to boundary conditions

$$
\Theta(x, y, t, u)=0
$$

holding on any fixed boundary in the problem (these may also include decay conditions on $u$ for large $|x|,|y|)$, the free surface $S$ is determined by a number of differential equations

$$
\Xi(x, t, \eta, u)=0
$$

holding on $S$ and involving $x, t, u$ and its derivatives evaluated on $S$, and $\eta$ and its derivatives with respect to $x$ and $t$. Often, as in the case of irrotational fluid flow, equations (3.3)-(3.4) will not involve $t$ or temporal derivatives of $u$, in which case, as detailed in [4], the time evolution can be thought of as taking place exclusively on $S$ with (3.3)-(3.4) constituting an additional boundary value problem to be integrated at each time so as to determine the values of the derivatives of $u$ on $S$. Generalizations to situations where the free surface folds over, and must be described parametrically, can, as discussed in [4], be readily incorporated into the theory. 
A distinction must always be maintained between a function $F(x, y, t, u)$ defined over $\Omega$ and its value on the free surface $S$, which is denoted by

$$
F_{S}(x, t, u)=F(x, \eta(x, t), t, u .)
$$

Certain formulae will hold over all of $\Omega$, while others only hold on the free surface. Often, to avoid unnecessary cluttering with $S$-subscripts, the latter will be indicated by the phrase "on $S$ " appended to them. It is also important to distinguish between two types of differentiation - that taking place in the region $\Omega$, denoted by usual subscripts, and that taking place on the free surface $S$, denoted by boldface subscripts. For instance

$$
u_{\mathbf{x}}=u_{x}+\eta_{\mathbf{x}} u_{y} \quad \text { on } S
$$

for $u=u(x, y, t)$ defined in $\Omega$. In $\S 6$ it will be seen that for the water wave problem, the function $\eta(x, t)$ itself can be thought of as the value on the free surface of a function $\eta(x, y, t)$ defined over all of $\Omega$; for this reason it is important to maintain the distinction between these two modes of differentiation even for $\eta$. Total derivatives are denoted by capital $D$ 's, boldface or not, depending on whether or not this takes place on the free surface. Note that for functions defined in $\Omega$,

$$
\mathbf{D}_{\mathbf{x}}=D_{x}+\eta_{\mathbf{x}} D_{y}, \quad \mathbf{D}_{\mathbf{t}}=D_{t}+\eta_{\mathbf{t}} D_{y} \quad \text { on } S
$$

so that, for example,

$$
\mathbf{D}_{\mathbf{x}}\left(u^{2}\right)=2 u u_{\mathbf{x}}=\left(D_{x}+\eta_{\mathbf{x}} D_{y}\right)\left(u^{2}\right)=2 u\left(u_{x}+\eta_{\mathbf{x}} u_{y}\right) \text { on } S .
$$

To understand how the notion of a conservation law is to be properly adapted to the situation of a free boundary problem, it is helpful to begin by discussing a simple example. For the water wave problem, assuming unit density, the mass

$$
M=\int_{S} \eta d x
$$

has the time variation

$$
\frac{d M}{d t}=\int_{S} \eta_{\mathbf{t}} d x=\int_{S}\left(\varphi_{y}-\eta_{\mathbf{x}} \varphi_{x}\right) d x=\int_{S} \varphi_{y} d x-\varphi_{x} d y,
$$

where we have used the usual notation for line integrals in the plane. Since $\varphi$ is harmonic, $\varphi_{y} d x-\varphi_{x} d y$ is a closed differential. Therefore, by Green's theorem,

$$
\frac{d M}{d t}=-\int_{\Gamma}\left(\varphi_{y} d x-\varphi_{x} d y\right),
$$

expressing the self-evident fact that the rate of change of mass is equal to the integral of the (outward) normal component of the velocity over the fixed boundary $\Gamma$.

In general, a conservation law will be such that its rate of change over any bounded region equals the total corresponding flux over the boundary of that region. Moreover, since the free surface moves with the fluid, this flux can take place only on the fixed component of the boundary which lies within the volume occupied by the fluid. Since we are interpreting the time evolution of the system as taking place on the free surface (subject to the auxiliary conditions (3.3)-(3.4)), the conserved 
quantity will be an integral $Q=\int_{S} T d x$ over the free surface, where the conserved density $T$ is defined just on the free surface itself. The conservation law is local if $T$ depends on $x, t, \eta$ and its derivatives and $u$ and its derivatives (evaluated on $S$ ) at each point of $S$-these are the only types of conservation laws to be considered here.

Consider a bounded domain $D \subset \mathbf{R}^{2}$, and let $\Omega \subset D$ be the part of $D$ filled with fluid at time $t$. The boundary of $\Omega$ consists of two pieces: the component $S$ of the free surface bounding $\Omega$ and a fixed component $\Gamma=\partial \Omega \cap \partial D$ within the total volume occupied by the fluid. The boundary of $S$, which is $\partial S=S \cap \Gamma$, consists of a finite number of points. Since $S$ moves with the fluid, flux can only occur at $\Gamma$ and $\partial S$. Therefore, the total flux, i.e. the time derivative of $Q$, must be expressed as the sum of a line integral over the fixed boundary and, possibly, a finite sum over the points of $\partial S$. In order that $d Q / d t$ be equal to a sum of fluxes over $\Gamma$ and over $\partial S$, it is necessary and sufficient that the form $D_{t} T d x$ be expressible as the sum of a closed differential defined over the region $\Omega$ and a total $x$-derivative. Thus if $\mathbf{D}_{\mathbf{t}} T=U-$ $\eta_{\mathbf{x}} V+\mathbf{D}_{\mathbf{x}} W$ on $S$ where $U, V$ are defined over $\Omega$ and $W$ is defined on $S$, then

$$
\begin{aligned}
\frac{d}{d t} \int_{S} T d x & =\int_{S}(U d x-V d y)+\int_{S} \mathbf{D}_{\mathbf{x}} W d x \\
& =\int_{\Gamma}(U d x-V d y)+\sum_{\partial S} W
\end{aligned}
$$

provided $U d x-V d y$ is closed. (Here the summation over $\partial S$ has appropriate signs attached to each point.) We are thus led to the following definition.

Definition 3.1. A function $T$ depending on $x, t, \eta, u$ and the derivatives of $\eta$ and $u$ over the free surface $S$ is a conserved density for the free boundary problem (3.3)-(3.5) if there exist functions $U, V$ depending on $x, y, t, u$ and the derivatives of $u$ in the region $\Omega$ and a function $W$ depending on $x, t, \eta, u$ and their derivatives on $S$ such that for all solutions $u=f(x, y, t), \eta=g(x, t)$ of the free boundary problem

$$
\mathbf{D}_{\mathbf{t}} T=U-\eta_{\mathbf{x}} V+\mathbf{D}_{\mathbf{x}} W \text { on } S,
$$

with

$$
D_{y} U+D_{x} V=0 \text { in } \Omega \text {. }
$$

If $T$ is a conserved density, then formula (3.7), equating the rate of change of the total density in terms of the total flux (as defined by $U, V, W$ ), will hold for all solutions of the free boundary problem.

Note that conditions (3.8)-(3.9) include, but are more general than, the naive condition

$$
\mathbf{D}_{\mathbf{t}} T=\mathbf{D}_{\mathbf{x}} W \text { on } S
$$

arising from a direct analogy with partial differential equations. Although the introduction of a stream function will put the conservation of mass result for the water wave problem in the above form, other examples discussed in [4] and the general characterization theorem of $\S 5$ show that it is overly restrictive to take (3.10) as the definition of conservation law. 
If the density $T$ can itself be written as the sum of a closed differential and a surface $x$-derivative, i.e.

$$
T=\tilde{U}-\eta_{\mathbf{x}} \tilde{V}+\mathbf{D}_{\mathbf{x}} \tilde{W} \text { on } S,
$$

with $D_{y} \tilde{U}+D_{x} \tilde{V}=0$ in $\Omega$, for all solutions of the free boundary problem, then clearly $T$ is conserved. Indeed (3.8) holds with

$$
U=D_{t} \tilde{U}, \quad V=D_{t} \tilde{V}, \quad W=\mathbf{D}_{\mathbf{t}} \tilde{W}-\eta_{\mathbf{t}} \tilde{V}
$$

Such densities are called trivial, and two densities will be called equivalent if their difference is a trivial density. Since we are only interested in nontrivial densities, it is of great importance to know when a given density is trivial.

For problems in higher dimensions the definition is similar. If $x=\left(x_{1}, \ldots, x_{p}\right)$ then the free surface $S$ and the fixed boundary $\Gamma$ will be $p$-dimensional submanifolds of $\mathbf{R}^{p+1}$, with intersection $\partial S$ a $(p-1)$ dimensional submanifold. For $T$ to be a conserved density, we require the existence of functions $U, \mathbf{V}=\left(V_{1}, \ldots, V_{p}\right)$ defined in $\Omega$ and $\mathbf{W}=\left(W_{1}, \ldots, W_{p}\right)$ defined on $S$ such that

$$
\mathbf{D}_{\mathbf{t}} T=U-\nabla \eta \cdot \mathbf{V}+\operatorname{Div} \mathbf{W} \text { on } S .
$$

Stokes' theorem then shows that

$$
\frac{d}{d t} \int_{S} T d x_{1} \cdots d x_{p}=\int_{\Gamma} \omega+\int_{\partial S} \mu
$$

where

$$
\begin{aligned}
\omega= & V_{1} d y \wedge d x_{2} \wedge \cdots \wedge d x_{p}+\cdots+V_{p} d y \wedge d x_{1} \wedge \cdots \wedge d x_{p-1} \\
& -U d x_{1} \wedge \cdots \wedge d x_{p}
\end{aligned}
$$

and

$$
\mu=W_{1} d x_{2} \wedge \cdots \wedge d x_{p}-\cdots \pm W_{p} d x_{1} \wedge \cdots \wedge d x_{p-1}
$$

again expressing the fact that the rate of change in total density equals the sum of the fluxes over the fixed boundary $\Gamma$ and the boundary of the free surface $\partial S$.

4. Volume and surface derivatives in harmonic free boundary problems. By abuse of language, a free boundary problem of the general form of $\$ 3$ will be called harmonic if the field functions $u$ consist just of one potential function $\varphi(x, y, t)$, and the constitutive equation (3.3) is just Laplace's equation

$$
\Delta \varphi=0 \text { in } \Omega \text {. }
$$

From here on, we restrict attention to the two-dimensional case (i.e. $x \in \mathbf{R}$ ) but we leave the form of the boundary conditions open for the time being.

Introduce the harmonic conjugate $\psi$ to $\varphi$, the stream function, so that the Cauchy-Riemann equations

$$
\varphi_{x}=\psi_{y}, \quad \varphi_{y}=-\psi_{x}
$$

are satisfied. Let

$$
\varphi_{n}^{m}=D_{t}^{m} D_{x}^{n} \varphi, \quad \psi_{n}^{m}=D_{t}^{m} D_{x}^{n} \psi
$$


so that $\varphi_{n}^{m}$ and $\psi_{n}^{m}$ are also harmonic conjugates, satisfying

$$
\begin{array}{ll}
D_{x} \varphi_{n}^{m}=\varphi_{n+1}^{m}, & D_{x} \psi_{n}^{m}=\psi_{n+1}^{m}, \\
D_{y} \varphi_{n}^{m}=-\psi_{n+1}^{m}, & D_{y} \psi_{n}^{m}=\varphi_{n+1}^{m} .
\end{array}
$$

We are thus justified in introducing the complex potentials

$$
\omega_{n}^{m}=\varphi_{n}^{m}+i \psi_{n}^{m}
$$

satisfying

$$
D_{z} \omega_{n}^{m}=\omega_{n+1}^{m}, \quad D_{\bar{z}} \omega_{n}^{m}=0 .
$$

Here the complex derivatives are given by

$$
D_{z}=\frac{1}{2}\left(D_{x}-i D_{y}\right), \quad D_{\bar{z}}=\frac{1}{2}\left(D_{x}+i D_{y}\right) .
$$

When $m=0$, we usually drop the extra superscripts, so $\omega_{n}=\omega_{n}^{0}$, etc.

As discussed in $\S 3$, for a free boundary problem there are two types of derivatives - volume and surface. In this section we derive important formulae relating the volume derivatives $\omega_{n}$ of the complex potential to its surface derivatives $\omega_{n}=\mathbf{D}_{\mathbf{x}}^{n} \omega=$ $\varphi_{\mathrm{n}}+i \psi_{\mathrm{n}}$. To begin, we introduce some differential polynomials of independent interest.

Let $u(x), v(x)$ be smooth, complex valued functions of a real variable $x$, with $u=d v / d x$, and $u_{n}=d^{n} u / d x^{n}$. Define $Y_{n}^{k}(u)$ to be the coefficient of the derivative $F^{(k)}(v)$ in the Faà-di-Bruno formula

$$
\frac{d^{n}}{d x^{n}}(F(v(x)))=\sum_{k=1}^{n} Y_{n}^{k}(u) F^{(k)}(v) .
$$

The polynomials $Y_{n}^{k}$ are known as the Bell polynomials [23] and have the general formula

$$
Y_{n}^{k}(u)=\sum \frac{n !}{I !} u^{i_{1}}\left(\frac{u_{1}}{2 !}\right)^{i_{2}} \cdots\left(\frac{u_{n-1}}{n !}\right)^{i_{n}}
$$

the sum being over all multi-indices $I=\left(i_{1}, \ldots, i_{n}\right), i_{\nu} \geqslant 0$, with $i_{1}+\cdots+i_{n}=k$, $i_{1}+2 i_{2}+\cdots+n i_{n}=n$. In particular,

$$
Y_{n}^{n}(u)=u^{n}, \quad Y_{n}^{1}(u)=u_{n-1} .
$$

Similarly, define $X_{n}^{k}(u)$ to be the coefficient of $F^{(k)}(x)$ in the formula

$$
\left(u \frac{d}{d x}\right)^{n} F(x)=\sum_{k=1}^{n} X_{n}^{k}(u) F^{(k)}(x) .
$$

The polynomials $X_{n}^{k}$ have been studied by Comtet in [7], in which explicit formulae and recursion relations for them are found. Here we need only note the special case

$$
X_{n}^{n}(u)=u^{n}
$$


Proposition 4.1. Let

$$
\zeta=1+i \eta_{\mathbf{x}}, \quad \theta=1 / \zeta .
$$

Then the volume and surface derivatives of the complex potential $\omega$ are related by the formulae

$$
\begin{aligned}
& \omega_{\mathbf{n}}=\sum_{k=1}^{n} Y_{n}^{k}(\zeta) \omega_{k}, \\
& \omega_{n}=\sum_{k=1}^{n} X_{n}^{k}(\theta) \omega_{\mathbf{k}},
\end{aligned}
$$

valid for $n \geqslant 1$. (In the polynomials $Y_{n}^{k}, X_{n}^{k}$, the derivatives of $\zeta, \theta$ used are the surface derivatives $\zeta_{n}, \theta_{n}$.)

Proof. Note first from (3.6) since $\varphi_{k}$ and $\psi_{k}$ are harmonic conjugates

$$
\mathbf{D}_{\mathbf{x}} \omega_{k}=\zeta \omega_{k+1} \text {. }
$$

Therefore,

$$
\omega_{\mathbf{n}+\mathbf{1}}=\sum_{k=1}^{n}\left[\left(\mathbf{D}_{\mathbf{x}} Y_{n}^{k}\right) \omega_{k}+\zeta Y_{n}^{k} \omega_{k+1}\right]=\sum_{k=1}^{n+1}\left(\mathbf{D}_{\mathbf{x}} Y_{n}^{k}+\zeta Y_{n}^{k-1}\right) \omega_{k} .
$$

The recursion relation for $Y_{n}^{k}$ is

$$
\mathbf{D}_{\mathbf{x}} Y_{n}^{k}(\zeta)+\zeta Y_{n}^{k-1}(\zeta)=Y_{n+1}^{k}(\zeta)
$$

hence (4.11) is established by induction. Similarly, applying $\mathbf{D}_{\mathbf{x}}$ to (4.12),

$$
\zeta \omega_{n+1}=\sum_{k=1}^{n}\left[\left(\mathbf{D}_{\mathbf{x}} X_{n}^{k}\right) \omega_{\mathbf{k}}+X_{n}^{k} \omega_{\mathbf{k}+1}\right]
$$

hence

$$
\omega_{n+1}=\sum_{k=1}^{n+1}\left[\theta \mathbf{D}_{\mathbf{x}} X_{n}^{k}+\theta X_{n}^{k-1}\right] \omega_{\mathbf{k}}
$$

which proves (4.12) by induction using an obvious recursion formula for the $X_{n}^{k}$.

In particular, from (4.7)-(4.9), the highest order terms occurring in (4.11)-(4.12) are

$$
\omega_{n}=\zeta^{n} \omega_{n}+\zeta_{n-1} \omega_{1}+\cdots
$$

and

$$
\omega_{n}=\theta^{n} \omega_{n}+\theta^{n-1} \theta_{n-1} \omega_{1}+\cdots=\zeta^{-n} \omega_{n}-\zeta^{-n-1} \zeta_{n-1} \omega_{1}+\cdots
$$

If we let

$$
\zeta^{n}=a_{n}+i b_{n}, \quad \theta^{n}=\zeta^{-n}=c_{n}+i d_{n},
$$

so that $a_{n}, b_{n}, c_{n}, d_{n}$ are real valued functions of $\eta_{\mathrm{x}}$ only, then the real and imaginary parts of (4.14)-(4.15) yield the formulae

$$
\begin{aligned}
& \varphi_{\mathrm{n}}=a_{n} \varphi_{n}-b_{n} \psi_{n}-\eta_{\mathrm{n}-1} \psi_{x}+\cdots, \\
& \psi_{\mathrm{n}}=b_{n} \varphi_{n}+a_{n} \psi_{n}+\eta_{\mathrm{n}-1} \varphi_{x}+\cdots,
\end{aligned}
$$


and

$$
\begin{aligned}
& \varphi_{n}=c_{n} \varphi_{\mathrm{n}}-d_{n} \psi_{\mathrm{n}}-\eta_{\mathrm{n}-\mathbf{1}}\left(d_{n+1} \varphi_{\mathbf{x}}+c_{n+1} \psi_{\mathbf{x}}\right)+\cdots, \\
& \psi_{n}=d_{n} \varphi_{\mathrm{n}}+c_{n} \psi_{\mathrm{n}}+\eta_{\mathrm{n}-\mathbf{1}}\left(c_{n+1} \varphi_{\mathbf{x}}-d_{n+1} \psi_{\mathbf{x}}\right)+\cdots .
\end{aligned}
$$

These formulae hold for any pair $\varphi, \psi$ of harmonically conjugate variables and will be of great use in the proof of the main theorem.

We conclude this section by reexpressing the free surface conditions (1.3)-(1.4) of the water wave problem entirely in terms of surface derivatives. We first have

$$
\varphi_{\mathrm{t}}=\varphi_{t}+\eta_{\mathrm{t}} \varphi_{y}=-\frac{1}{2}|\nabla \varphi|^{2}+\eta_{\mathrm{t}} \varphi_{y}-g \eta=\frac{1}{2} \varphi_{y}^{2}-\frac{1}{2} \varphi_{x}^{2}-\eta_{\mathrm{x}} \varphi_{x} \varphi_{y}-g \eta,
$$

which was used in [4] to find the Hamiltonian form of the water wave problem. However, we can further use (4.12) to reexpress this:

$$
\varphi_{\mathrm{t}}=\left(1+\eta_{\mathrm{x}}^{2}\right)^{-1}\left(\frac{1}{2} \psi_{\mathrm{x}}^{2}-\frac{1}{2} \varphi_{\mathrm{x}}^{2}-\eta_{\mathrm{x}} \varphi_{\mathrm{x}} \psi_{\mathrm{x}}\right)-g \eta, \quad \eta_{\mathrm{t}}=-\psi_{\mathrm{x}} \quad \text { on } S
$$

5. A characterization of conserved densities of two-dimensional harmonic problems. For a two-dimensional harmonic free boundary problem, the general conditions (3.8)-(3.9) for a conserved density can, by elementary techniques from complex analysis, be simplified. Note that the coefficients $U, V$ of the exact differential in (3.7) are now functions of $x, y, \varphi_{n}^{m}, \psi_{n}^{m}$. The basic characterization theorem then states that either $U, V$ arise from a total $\mathbf{x}$-derivative along the free surface $S$, or they are the real and imaginary parts of an analytic function of the complex variables $z=x+i y$ and $\omega_{n}^{m}$. Specifically:

THEOREM 5.1. The function $T$ is a conserved density for a two-dimensional harmonic free boundary problem, if and only if there is a complex analytic function $Z=X+i Y$ depending on the complex variables $z, \omega_{n}^{m}, m, n \geqslant 0$, and a real valued function $W$ defined on the free surface $S$ satisfying

$$
\mathbf{D}_{\mathbf{t}} T=\operatorname{Re}(\zeta Z)+\mathbf{D}_{\mathbf{x}} W=\left(X+D_{x} W\right)+\eta_{\mathbf{x}}\left(-Y+D_{y} W\right),
$$

where $\zeta=1+i \eta_{\mathbf{x}}$. In other words, only those closed differentials arising from analytic functions make nontrivial contributions to the flux over the fixed boundary $\Gamma$.

To prove this theorem, it suffices to show that (3.9) holds for functions $U, V$ of $x, y, \varphi_{n}^{m}, \psi_{n}^{m}$ if and only if $U=X+D_{x} W, V=Y-D_{y} W$ for $X, Y, W$ satisfying the conditions of the theorem. A priori, it is somewhat surprising to find just one-half of the "total derivative" Cauchy-Riemann equations for $U, V$ sufficient to ensure analyticity up to a potential function $W$, but this is indeed the case.

Note that the theorem also serves to characterize all trivial conserved densities. That is, $T$ is trivial if and only if

$$
T=\operatorname{Re}(\zeta Z)+\mathbf{D}_{\mathbf{x}} W
$$

for $Z, W$ as above. This will be important in the classification of conservation laws for the water wave problem.

For the next two lemmas, $z=x+i y$ and $w=u+i v$ will denote independent complex variables. 
LEMMA 5.2. Suppose $f$ and $g$ are complex valued functions of $z$ and $w$ which are analytic in $z$, but not necessarily analytic in $w$. Then

$$
\operatorname{Im}\left(\bar{z} \partial_{\bar{w}} f+g\right)=0
$$

(where $\partial_{\bar{w}}=\partial / \partial \bar{w}$, etc.) if and only if

$$
f=h(z, w)+z \partial_{w} \alpha(u, v)+\beta(u, v), \quad g=z \partial_{w} \bar{\beta},
$$

where $h$ is analytic in both $z$ and $w, \alpha$ is a real valued function of $u, v$ and $\beta$ is any complex-valued function of $u, v$. Moreover, $h, \alpha, \beta$ are uniquely determined by the conditions $h(0, w)=0=\partial_{z} h(0, w)$.

Proof. Let $f=a+i b, g=c+i d$, where $a, b, c, d$ are real valued functions. Then (5.3) reads

$$
\frac{1}{2} x\left(a_{v}+b_{u}\right)-\frac{1}{2} y\left(a_{u}-b_{v}\right)+d=0 .
$$

Now $a, b, c, d$ are all harmonic in $x, y$; hence, applying the Laplacian $\partial_{x}^{2}+\partial_{y}^{2}$ to the above equation we find $a_{x v}+b_{x u}+b_{y v}-a_{y u}=0$. This simplifies to

$$
a_{x v}=a_{y u} \text {, }
$$

since $a$ and $b$ are conjugate harmonic in $x, y$. This in turn implies that $a_{x x}, b_{x x} ; a_{x y}$, $b_{x y}$; and $a_{y y}, b_{y y}$ all satisfy the Cauchy-Riemann equations in $u, v$; hence

$$
\partial_{z}^{2} f=k(z, w),
$$

where $k$ is analytic in both $z$ and $w$. Then

$$
f(z, w)=\int_{0}^{z}\left(z-z^{\prime}\right) k\left(z^{\prime}, w\right) d z^{\prime}+z \gamma(u, v)+\beta(u, v)
$$

where $\gamma$ and $\beta$ are complex valued. Finally, (5.4) implies that $\gamma=\partial_{w} \alpha$ for some real valued $\alpha$, completing the proof.

LemMa 5.3. Suppose $\alpha(x, y ; u, v)$ is real valued. Then $\partial_{w} \alpha$ is analytic in $z$ if and only if $\partial_{w} \alpha=h(z, w)+\partial_{w} \beta$, where $h$ is analytic in both $z$ and $w$, and $\beta$ is real valued and depends only on $u, v$.

Proof. The Cauchy-Riemann equations in $x, y$ for $\partial_{w} \alpha \operatorname{read} \alpha_{x u}=-\alpha_{y v}, \alpha_{y u}=$ $\alpha_{x v}$. Differentiation with respect to $u$ and $v$ shows that $\alpha_{x}$ and $\alpha_{y}$ are both harmonic in $u, v$, hence $\alpha=\gamma(x, y ; u, v)+\beta(u, v)$, where $\gamma$ is harmonic in $u, v, \gamma(0,0 ; u, v)$ $=0$. Then $\partial_{w} \gamma$ is analytic in $w$, and the lemma follows.

It clearly suffices to prove Theorem 5.1 for a single series $\omega_{n}=\varphi_{n}+i \psi_{n}$ of harmonically conjugate variables, so we drop the extra index $m$. The theorem is then implied by the following proposition.

Proposition 5.4. Suppose $U$ and $V$ depend on $x, y$ and the harmonic conjugate variables $\varphi_{n}, \psi_{n}$, which satisfy (4.3). Then

$$
D_{y} U+D_{x} V=0
$$

if and only if

$$
U=X+D_{x} W, \quad V=Y-D_{y} W,
$$


where $Z=X+i Y$ is an analytic function of $z=x+i y, \omega_{n}=\varphi_{n}+i \psi_{n}$ and $W$ is arbitrary. Moreover $Z$ and $W$ are uniquely specified by the additional conditions

$$
\left.\partial_{\omega_{n}} Z\right|_{0}=0, \quad n=1,2,3, \ldots,
$$

where $\left.\right|_{0}$ denotes evaluation at $\omega_{k}=0$ for $k \geqslant n$.

Proof. Let $\Theta=U+i V$, so that (5.6) can be rewritten as $\Theta=Z+2 D_{z} W$. Suppose by reverse induction on $n$ we have established that $\Theta=Z^{n}+2 D_{z} W^{n}$, where $Z^{n}$ is analytic in $\omega_{k}$ for $k \geqslant n$ and uniquely determined by the conditions $\left.\partial_{\omega_{k}} Z^{n}\right|_{0}=0, k \geqslant n+1$. Since $\varphi_{n}, \psi_{n}$ are harmonically conjugate,

$$
D_{\bar{z}}=\partial_{\bar{z}}+\sum_{i=0}^{\infty} \bar{\omega}_{i+1} \partial_{\bar{\omega}_{i}} ;
$$

hence

$$
D_{\bar{z}} Z^{n}=\bar{\omega}_{n} \partial_{\bar{\omega}_{n-1}} Z^{n}+D_{\bar{z}}^{0} Z^{n}
$$

where

$$
D_{\bar{z}}^{0}=\partial_{\bar{z}}+\sum_{i=0}^{n-2} \bar{\omega}_{i+1} \partial_{\bar{\omega}_{i}} .
$$

Then (5.5) can be written as

$$
0=\operatorname{Im}\left(D_{\bar{z}} \Theta\right)=\operatorname{Im}\left(\bar{\omega}_{n} \partial_{\bar{\omega}_{n-1}} Z^{n}+D_{\bar{z}}^{0} Z^{n}\right)
$$

since $D_{\bar{z}} D_{z} W^{n}$ is real. Lemma 5.2 implies that

$$
Z^{n}=\tilde{Z}^{n-1}+\omega_{n} \partial_{\omega_{n-1}} \tilde{A}^{n}+\tilde{B}^{n}
$$

where $\tilde{Z}^{n-1}$ is analytic in $\omega_{k}, k \geqslant n-1, \tilde{A}^{n}$ is real valued, and $\tilde{A}^{n}$ and $\tilde{B}^{n}$ are independent of $\varphi_{n}, \psi_{n} ; Z^{n-1}, \tilde{A}^{n}, \tilde{B}^{n}$ are uniquely determined by the conditions

$$
\left.\tilde{Z}^{n-1}\right|_{\omega_{n}=0}=0=\left.\partial_{\omega_{n}} \tilde{Z}^{n-1}\right|_{\omega_{n}=0} .
$$

Next apply $\partial_{\omega_{n}}$ to (5.8) to find

$$
\partial_{\omega_{n-1}} \tilde{A}^{n}=\left.\partial_{\omega_{n}} Z^{n}\right|_{\omega_{n}=0}
$$

so by Lemma 5.3,

$$
\partial_{\omega_{n-1}} \tilde{A}^{n}=\hat{Z}^{n-1}+\partial_{\omega_{n-1}} A^{n}
$$

where $\hat{Z}^{n-1}$ is analytic in $\omega_{k}$ for $k \geqslant n-1$, and $A^{n}$ does not depend on $\omega_{k}$ for $k \geqslant n$; these are also uniquely determined by the condition that $\hat{Z}^{n-1}=0$ when $\omega_{k}=0$ for $k \geqslant n$. Therefore

$$
Z^{n}=Z^{n-1}+D_{z} W^{n-1}+B^{n}
$$

where $Z^{n-1}=\tilde{Z}^{n-1}+\omega_{n} \hat{Z}^{n-1}$, and $W^{n-1}, B^{n}$ are determined from $A^{n}, \tilde{B}^{n}$.

Finally, to eliminate $B^{n}$ in the expression for $Z^{n}$ and so complete the induction step, note that

$$
0=\operatorname{Im} D_{\bar{z}} Z^{n}=\operatorname{Im}\left(\bar{\omega}_{n} \partial_{\bar{\omega}_{n-1}} B^{n}+C^{n}\right),
$$


where $C^{n}=D_{\bar{z}}^{0}\left(Z^{n-1}+B^{n}\right)$. Again using Lemma 5.2, we find

$$
C^{n}=\omega_{n} \partial_{\omega_{n-1}} \overline{B^{n}}
$$

hence

$$
\overline{\partial_{\bar{\omega}_{n-1}} B^{n}}=\partial_{\omega_{n}} D_{\bar{z}}^{0}\left(Z^{n-1}+B^{n}\right)=D_{\bar{z}}\left(\partial_{\omega_{n}} Z^{n-1}\right)=0,
$$

when $\omega_{k}=0$ for $k \geqslant n$. Therefore $\partial_{\bar{\omega}_{n-1}} B^{n}=0$, so $B^{n}$ is actually analytic in $\omega_{n-1}$ and can be incorporated into $Z^{n-1}$.

It remains to discuss the dependence on $x$ and $y$. Now if $\Theta=Z^{0}+D_{z} W^{0}$, with $Z^{0}=X^{0}+i Y^{0}$ analytic in $\omega_{n}$ for all $n,(5.5)$ implies that $\partial_{y} X^{0}+\partial_{x} Y^{0}=0$. Thus $Z^{0}=\partial_{z} A^{0}$ for some real valued $A^{0}$. Lemma 5.3 then proves that

$$
Z^{0}=Z+\partial_{z} \beta(x, y), \quad \beta \text { real, }
$$

which completes the proof of the proposition.

6. Formulations of the water wave problem. To correctly distinguish trivial from nontrivial conservation laws, it is of primary importance to determine a complete set of independent variables and to describe the system in terms of these variables. In this paper, we will take a somewhat unconventional view of the nature of the free boundary problem for water waves. The motivation for this viewpoint is the recurrent difficulty in any attempt to classify conservation laws that the function $\eta(x, t)$ describing the free surface is only defined as a function of the horizontal coordinate $x$ and time $t$, whereas the potential function $\varphi$ is defined over the entire domain $\Omega$. We propose to obviate this difficulty by extending the domain of definition of $\eta$ from the free surface $S$ to the entire domain $\Omega$.

The dynamical surface condition

$$
\varphi_{t}+\frac{1}{2}|\nabla \varphi|^{2}+g \eta=0 \text { on } S
$$

arises from Bernoulli's equation, given vanishing of the pressure on the free surface; within the body of the fluid, Bernoulli's equation reads

$$
\varphi_{t}+\frac{1}{2}|\nabla \varphi|^{2}+g y=p / \rho \text { in } \Omega
$$

where $p$ denotes pressure and $\rho$ the density of the fluid. (An arbitrary function of $t$ which would ordinarily appear [12] has been normalized to zero through the dynamical surface condition.) Comparing these two versions of Bernoulli's equation, it seems reasonable to define the function $\eta(x, y, t)$ over the domain $\Omega$ as the variable part of the pressure; in other words,

$$
\eta=y+p / g \rho=-g^{-1}\left(\varphi_{t}+\frac{1}{2}|\nabla \varphi|^{2}\right) \quad \text { in } \Omega .
$$

The dynamical free surface condition is now replaced by the implicit relation

$$
\eta(x, y, t)=y \quad \text { on } S,
$$

defining $y$ as a function of $x, t$, namely $y=\eta(x, t)=\left.\eta(x, y, t)\right|_{s}$.

Formula (6.1) permits us to define volume derivatives $\eta_{x}, \eta_{y}, \eta_{t}$, etc. of $\eta$ in terms of volume derivatives of $\varphi$. On $S$, formulae (3.6) for the surface derivatives lead to the relations

$$
\eta_{\mathbf{x}}=\eta_{x} /\left(1-\eta_{y}\right), \quad \eta_{\mathbf{t}}=\eta_{t} /\left(1-\eta_{y}\right)
$$


for the surface derivatives of $\eta$ in terms of its volume derivatives. In (6.3) we can further substitute for the volume derivatives according to (6.1). The spatial derivative becomes

$$
\eta_{\mathbf{x}}=\frac{\varphi_{x t}+\varphi_{x} \varphi_{x x}+\psi_{x} \psi_{x x}}{\psi_{x t}+\varphi_{x} \psi_{x x}-\psi_{x} \varphi_{x x}-g}=\frac{\varphi_{x t}+\alpha}{\psi_{x t}+\beta},
$$

where $\psi$ is the stream function and

$$
\alpha=\varphi_{x} \varphi_{x x}+\psi_{x} \psi_{x x}, \quad \beta=\varphi_{x} \psi_{x x}-\psi_{x} \varphi_{x x}-g .
$$

Substituting the corresponding expression for the temporal derivative $\eta_{t}$ into the kinematic boundary condition (1.4), we are led to an equation for the second time derivative of $\varphi$ solely in terms of $\varphi$ and $\psi$ :

$$
\begin{aligned}
\varphi_{t t} & =F_{0}^{2}\left(\varphi_{x t}, \psi_{x t}, \varphi_{x}, \psi_{x}, \varphi_{x x}, \psi_{x x}\right) \\
& =-2 \varphi_{x} \varphi_{x t}-2 \psi_{x} \psi_{x t}+\left(\psi_{x}^{2}-\varphi_{x}^{2}\right) \varphi_{x x}-2 \varphi_{x} \psi_{x} \psi_{x x}+g \psi_{x} \text { on } S .
\end{aligned}
$$

In our formulation, then, a solution to the water wave problem is given by a harmonic function $\varphi(x, y, t)$, defined in a domain $\Omega$ whose free boundary $y=\eta(x, t)$ is the implicit solution of $(6.2)(\eta(x, y, t)$ being given by (6.1)) satisfying boundary or decay conditions on fixed boundaries of $\Omega$, and the second order differential equation (6.6) on the free boundary. Note that parametrically described surfaces also fall within this formulation.

From this point of view, a complete set of independent variables for the water wave problem in the domain $\Omega$ is given by the collection

$$
\mathcal{V}_{\Omega}=\left\{x, y, t, \varphi_{n}^{m}, \psi_{n}^{m}: m \geqslant 0, n \geqslant 0\right\},
$$

where

$$
\varphi_{n}^{m} \equiv D_{t}^{m} D_{x}^{n} \varphi, \quad \psi_{n}^{m} \equiv D_{t}^{m} D_{x}^{n} \psi .
$$

In general, if $\mathcal{V}$ is any set of variables, $C(\mathcal{V})$ will denote the space of functions depending smoothly on the variables in $\mathfrak{T}$.

We will consistently use lexicographic ordering of the indices $(m, n)$, so that $(j, k)<(m, n)$ if $j<m$, or $j=m$ and $k<n$. Let

$$
\Upsilon_{\Omega}^{m, n}=\left\{x, y, t, \varphi_{k}^{j}, \psi_{k}^{j}:(j, k)<(m, n)\right\},
$$

and define $\mathcal{L}_{n}^{m}=C\left(\mathcal{V}_{\Omega}^{m, n}\right)$. Thus $F \in \mathcal{L}_{n}^{m}$ if and only if $F$ depends on variables $\varphi_{k}^{j}$, $\psi_{k}^{j}$ of order less than $(m, n)$.

On the free surface $S,(6.6)$ and its surface derivatives reduce the above collection of independent variables to

$$
\mathscr{V}_{S}=\left\{x, t, \varphi_{n}, \psi_{n}, \varphi_{n}^{1}, \psi_{n}^{1}, \psi_{n}^{m}: m \geqslant 2, n \geqslant 0\right\} .
$$

Indeed, since $\mathrm{D}_{\mathrm{t}} \varphi_{0}^{m}=\varphi_{0}^{m+1}-\eta_{t} \psi_{1}^{m}$, the $(m-2)$ nd surface t-derivative of (6.6) leads to an equation of the form

$$
\varphi_{0}^{m}=F_{0}^{m} \quad \text { on } S, \quad m \geqslant 2,
$$

where $F_{0}^{m} \in \mathcal{L}_{0}^{m}$. Taking surface x-derivatives of (6.9) leads to

$$
\varphi_{\mathrm{n}}^{m}=\mathbf{D}_{\mathbf{x}}^{n} F_{0}^{m} \equiv F_{\mathbf{n}}^{m} \quad \text { on } S, \quad m \geqslant 2, n \geqslant 0,
$$


where $F_{\mathbf{n}}^{m} \in \mathcal{L}_{0}^{m}$. Furthermore, (4.17) shows that

$$
\varphi_{n}^{m}=\left(b_{n} / a_{n}\right) \psi_{n}^{m}+F_{n}^{m} \text { on } S, \quad m \geqslant 2, n \geqslant 0,
$$

where $a_{n}, b_{n}$ are defined in (4.16) and $F_{n}^{m} \in \mathcal{L}_{n}^{m}$. Clearly no other relations between $\varphi_{n}^{m}$ and $\psi_{n}^{m}$ on $S$ exist, and hence the above collection is an independent set of variables. In particular, surface derivatives of $\eta$ can be found from surface derivatives of (6.4), using the definition (6.1) of $\eta$, and finally using (6.10) to eliminate higher order $t$-derivatives of $\varphi$. The exact form of the resulting expression will never be required here.

If $F \in C\left(\mathfrak{V}_{\Omega}\right)$, then $F$ will restrict on the free surface to a function $F_{S} \in C\left(\mathfrak{V}_{S}\right)$, which is found merely by substituting for the variables $\varphi_{n}^{m}, m \geqslant 2$, according to (6.9), (6.11) wherever they occur in $F$. In any equation holding on $S$ involving such a function, it is always assumed that such a substitution has been made. Let $\mathfrak{V}_{S}^{m, n} \subset \mathfrak{V}_{S}$ be the subset of independent surface variables obtained by restricting $\mathfrak{V}_{\Omega}^{m, n}$ to $S$. We will also write $\mathcal{L}_{n}^{m}$ for $C\left(\mathscr{V}_{S}^{m, n}\right)$.

7. The main theorem. We are now in a position to precisely state the main theorem on conservation laws of the water wave problem. Definition 3.1 says that a conserved density $T$ will be a smooth function, depending on the surface variables $\Upsilon_{S}$, which satisfies the equation

$$
\mathbf{D}_{\mathbf{t}} T=U-\eta_{\mathbf{x}} V+\mathbf{D}_{\mathbf{x}} W \text { on } S,
$$

where $W \in C\left(\mathscr{V}_{S}\right)$ is defined on $S$, whereas $U, V \in C\left(\mathscr{V}_{\Omega}\right)$ are defined over all of the fluid and satisfy the additional closure condition

$$
D_{y} U+D_{x} V=0 \text { in } \Omega \text {. }
$$

If $T$ itself is of this form,

$$
T=\tilde{U}-\eta_{\mathbf{x}} \tilde{V}+\tilde{W},
$$

$\tilde{U}, \tilde{V}, \tilde{W}$ as above, then (7.1) holds trivially. The goal is to classify all inequivalent nontrivial conserved densities, two densities being equivalent if their difference is trivial, i.e. of the form (7.3).

THEOREM 7.1. Let $T$ be a smooth function of the surface variables $\mathcal{V}_{S}$. Then $T$ is a conserved density for the water wave problem if and only if

$$
T=\sum_{i=1}^{8} c_{i} T_{i}+T_{0},
$$

where $c_{i}$ are constants, $T_{1}, \ldots, T_{8}$ are the conserved densities in (1.5) and $T_{0}$ is trivial.

In other words, the eight densities in the introduction are the only nontrivial ones. The remainder of this paper is devoted to a proof of this theorem. The key ingredient in the proof is the characterization theorem 5.1 for conservation laws of harmonic free boundary problems. It states that condition (7.1) can be replaced by the stronger condition

$$
\mathbf{D}_{\mathbf{t}} T=\operatorname{Re}(\zeta Z)+\mathbf{D}_{\mathbf{x}} W
$$


where $W \in C\left(\mathscr{V}_{S}\right)$ is real valued, while $Z \in C\left(\mathscr{V}_{\Omega}\right)$ is a complex valued analytic function of the variables $z=x+i y, \omega_{n}^{m}=\varphi_{n}^{m}+i \psi_{n}^{m}$. Uniqueness criteria for $Z$ and $W$ inherent in Proposition 5.4 will also occasionally be used to avoid duplication of characterizations of the same density.

The proof proceeds by eliminating nontrivial dependence of $T$ on the variables $\psi_{n}^{m}$ in descending lexicographic order. Then dependence on higher order surface $\mathbf{x}$-derivatives of $\varphi$ and, finally, higher order surface $\mathbf{x}$-derivatives of $\eta$ are in turn eliminated, so that we are left with nontrivial densities depending only on the first order variables $x, t, \varphi, \varphi_{\mathbf{x}}, \psi, \eta, \eta_{\mathbf{x}}$. The final calculation is somewhat eased by the form of the dependence on those last variables gleaned from previous reductions. At each stage, the basic idea of proof is the same, but the detailed computations necesarily change.

8. Discussion of variables. In the course of the proof of Theorem 7.1, a number of subsets of the collection of surface variable $\mathcal{V}_{S}$ arise, and it is convenient to list and briefly discuss them here.

(i) First order $t$-derivatives: $\quad \mathcal{V}_{S}^{t}=\left\{x, t, \varphi_{n}, \psi_{n}, \varphi_{n}^{1}, \psi_{n}^{1}: n \geqslant 0\right\}$.

(ii) Surface x-derivatives of $\varphi, \psi, \eta: \mathscr{T}_{S}^{\psi}=\left\{x, t, \varphi_{\mathrm{n}}, \psi_{\mathrm{n}}, \eta_{\mathrm{n}}: n \geqslant 0\right\}$.

(iii) Surface x-derivatives of $\varphi, \eta: \quad \mathcal{V}_{S}^{\varphi}=\left\{x, t, \varphi_{\mathbf{n}}, \eta_{\mathbf{n}}: n \geqslant 0\right\}$.

(iv) Surface x-derivatives of $\eta: \quad \mathcal{T}_{S}^{\eta}=\left\{x, t, \eta_{\mathrm{n}}: n \geqslant 0\right\}$.

Note that the first set of variables $\mathcal{V}_{S}^{t}$ is equivalent to the set $\overline{\mathcal{V}}_{S}^{t}=\left\{x, t, \varphi_{n}, \psi_{n}\right.$, $\left.\varphi_{\mathbf{n}}^{1}, \psi_{\mathbf{n}}^{1}: n \geqslant 0\right\}$. Indeed, to express $\varphi_{t x}, \psi_{t x}$ in terms of $\varphi_{t \mathbf{x}}, \psi_{t \mathbf{x}}$ we solve the equations

$$
\varphi_{t \mathbf{x}}=\varphi_{t x}-\eta_{\mathbf{x}} \psi_{t x}, \quad \psi_{t \mathbf{x}}=\psi_{t x}+\eta_{\mathbf{x}} \varphi_{t x}
$$

and (6.4) to find that

$$
\varphi_{t x}=\beta \delta\left(\varphi_{t \mathbf{x}} \psi_{t \mathbf{x}}+\beta \varphi_{t \mathbf{x}}+\alpha \psi_{t \mathbf{x}}\right), \quad \psi_{t x}=-\beta \delta\left(\left(\varphi_{t \mathbf{x}}\right)^{2}+\alpha \varphi_{t \mathbf{x}}-\beta \psi_{t \mathbf{x}}\right),
$$

where $\alpha, \beta$ are given by (6.5) and

$$
1 / \delta=\left(\varphi_{t \mathbf{x}}+\alpha\right)^{2}+\beta^{2} .
$$

In turn, (6.4) expresses $\eta_{\mathrm{x}}$ in terms of $\varphi_{t \mathrm{x}}, \psi_{t \mathrm{x}}$, and hence the surface derivatives $\eta_{\mathrm{n}}$ in terms of the surface derivatives $\varphi_{\mathbf{k}}^{1}, \psi_{\mathbf{k}}^{1}$ of $\varphi_{t}, \psi_{t}$. Finally, (4.12) gives the full expressions for $\varphi_{n}^{1}, \psi_{n}^{1}$ in terms of the surface derivatives $\varphi_{\mathbf{k}}^{1}, \psi_{\mathbf{k}}^{1}$. The explicit formulae will never be required here. A similar argument using (4.11) shows that the variables $\mathcal{V}_{s}^{\psi}$ are equivalent to $\overline{\mathcal{V}}_{s}^{\psi}=\left\{x, t, \varphi_{n}, \psi_{n}, \eta_{\mathrm{n}}: n \geqslant 0\right\}$.

The proof of Theorem 7.1 proceeds in four stages. At each stage, it is shown that the dependence of $T$ on variables not in the next subset is trivial. Thus, for instance, the first stage of the proof is to show that if $T \in C\left(\mathscr{V}_{S}\right)$, then $T \simeq \bar{T}+T_{0}$, where $\bar{T} \in C\left(\mathcal{V}_{S}^{t}\right)$ and $T_{0}$ is trivial. The essential feature of the water wave problem that permits us to proceed in this fashion is that the $t$-derivative of any density always depends on more variables than the density itself does. Analysis of the dependence of $\mathbf{D}_{\mathbf{t}} T$ on these additional variables allows us to conclude that the dependence of $T$ itself on the highest order variables is necessarily trivial. In the proof, however, this simple idea is somewhat obscured by the necessarily messy details of computation, for which I apologize in advance. 


\section{Higher order $t$-derivatives.}

LEMMA 9.1. Suppose $T \in C\left(\mathfrak{V}_{S}\right)$ is a conserved density for the water wave problem. Then

$$
T=\bar{T}+T_{0},
$$

where $\bar{T} \in C\left(\mathcal{V}_{S}^{t}\right)$ and $T_{0}$ is trivial.

Proof. The proof will be done by reverse lexicographic induction. Suppose $\psi_{n}^{m}$ $(m \geqslant 2)$ is the highest order derivative that $T$ depends on; in the notation of $\S 6$, $T \in \mathcal{L}_{n+1}^{m}$. Then by (4.18),

$$
\mathbf{D}_{\mathbf{t}} T=\sum_{j=0}^{n} T_{\psi_{j}^{m}} \psi_{j}^{m+1}+R_{1}=\sum_{j=0}^{n} T_{\psi_{j}^{m}}\left(d_{j} \varphi_{\mathrm{j}}^{m+1}+c_{j} \psi_{\mathrm{j}}^{m+1}\right)+R_{2},
$$

where $R_{1}, R_{2} \in \mathcal{L}_{0}^{m+1}$ and subscripts on $T$ denote partial derivatives. Integrating each summand by parts and using (6.9), we find for some well-defined $X$,

$$
\begin{aligned}
\mathbf{D}_{\mathbf{t}} T & =\left[\sum_{j=0}^{n}\left(-\mathbf{D}_{\mathbf{x}}\right)^{j}\left(c_{j} T_{\psi_{j}^{m}}\right)\right] \psi_{0}^{m+1}+\left[\sum_{j=0}^{n}\left(-\mathbf{D}_{\mathbf{x}}\right)^{j}\left(d_{j} T_{\psi_{j}^{m}}\right)\right] \varphi_{0}^{m+1}+R_{2}+\mathbf{D}_{\mathbf{x}} X \\
& =A \psi_{0}^{m+1}+R_{3}+\mathbf{D}_{\mathbf{x}} X
\end{aligned}
$$

where $A=\Sigma\left(-\mathbf{D}_{\mathbf{x}}\right)^{j}\left(c_{j} T_{\psi_{j}^{m}}\right)$ and $R_{3}$ are in $\mathcal{L}_{0}^{m+1}$. Next look at the highest order terms in $A$. Since each $c_{j} \in \mathcal{L}_{0}^{2}$, by (4.17),

$$
\begin{aligned}
A & =\left(-\mathbf{D}_{\mathbf{x}}\right)^{n}\left(c_{n} T_{\psi_{n}^{m}}\right)+B_{1}=(-1)^{n} c_{n} T_{\psi_{n}^{m} \psi_{n}^{m}}\left(\mathbf{D}_{\mathbf{x}}^{n} \psi_{n}^{m}\right)+B_{2} \\
& =(-1)^{n} c_{n} T_{\psi_{n}^{m} \psi_{n}^{m}}\left(b_{n} \varphi_{2 n}^{m}+a_{n} \psi_{2 n}^{m}\right)+B_{3},
\end{aligned}
$$

for certain $B_{j} \in \mathcal{e}_{2 n}^{m}, j=1,2,3,4$. By (6.11) we find that

$$
A=(-1)^{n} c_{n}\left(b_{n} \frac{b_{2 n}}{a_{2 n}}+a_{n}\right) T_{\psi_{n}^{m} \psi_{n}^{m}} \psi_{2 n}^{m}+B_{4}=(-1)^{n} \frac{a_{n}^{2}}{a_{n}^{2}-b_{n}^{2}} T_{\psi_{n}^{m} \psi_{n}^{m}} \psi_{2 n}^{m}+B_{4},
$$

where we have used the fact that

$$
a_{2 n}+i b_{2 n}=\zeta^{2 n}=\left(a_{n}+i b_{n}\right)^{2}, \quad c_{n}=a_{n} /\left(a_{n}^{2}+b_{n}^{2}\right) .
$$

If $T$ is to satisfy the identity (7.5), the term $A \psi_{0}^{m+1}$ in $\mathbf{D}_{\mathbf{t}} T$ can only arise from the contribution of the analytic function $Z$, hence $Z=Z^{\prime} \omega_{0}^{m+1}+Z_{0}$, where $Z^{\prime}, Z_{0} \in$ $\mathcal{E}_{0}^{m+1}$. Moreover the highest order terms in $A$ involve $\psi_{2 n}^{m}$ linearly, hence $Z^{\prime}=$ $(-1)^{n} Z^{\prime \prime} \omega_{2 n}^{m}+Z_{0}^{\prime}$, where $Z^{\prime \prime}, Z_{0}^{\prime} \in \mathcal{L}_{2 n}^{m}$. Then

$$
\begin{aligned}
Z & =(-1)^{n} i Z^{\prime \prime} \omega_{2 n}^{m} \psi_{0}^{m+1}+Z^{*} \\
& =(-1)^{n} Z^{\prime \prime}\left(-1+i b_{2 n} / a_{2 n}\right) \psi_{2 n}^{m} \psi_{0}^{m+1}+Z^{* *} \text { on } S
\end{aligned}
$$

for $Z^{*}, Z^{* *} \in \mathcal{L}_{2 n}^{m}$. Equating the highest order terms in $\mathbf{D}_{\mathbf{t}} T$ with the highest order terms in $\operatorname{Re}(\zeta Z)$, we are led to the condition

$$
T_{\psi_{n}^{m} \psi_{n}^{m}}=-\operatorname{Re}\left\{\left(1-i b_{n} / a_{n}\right)^{2} \zeta Z^{\prime \prime}\right\} \quad \text { on } S .
$$


Suppose $\hat{Z}$ is an analytic function satisfying

$$
\partial^{2} \hat{Z} /\left(\partial \omega_{n}^{m}\right)^{2}=Z^{\prime \prime} \quad \text { in } \Omega \text {. }
$$

Note that by (6.11),

$$
\frac{\partial}{\partial \psi_{n}^{m}}\left(\hat{Z}_{S}\right)=\left[\left(1-i \frac{b_{n}}{a_{n}}\right) \frac{\partial \hat{Z}}{\partial \psi_{n}^{m}}\right]_{S}=\left[\left(i+\frac{b_{n}}{a_{n}}\right) \frac{\partial \hat{Z}}{\partial \omega_{n}^{m}}\right]_{S} .
$$

Therefore

$$
\frac{\partial^{2}}{\left(\partial \psi_{n}^{m}\right)^{2}}\left(\hat{Z}_{S}\right)=\left[\left(i+\frac{b_{n}}{a_{n}}\right)^{2} \frac{\partial^{2} \hat{Z}}{\left(\partial \omega_{n}^{m}\right)^{2}}\right]_{S}=-\left(1-i \frac{b_{n}}{a_{n}}\right)^{2} Z_{S}^{\prime \prime}
$$

hence

$$
\partial^{2} T /\left(\partial \psi_{n}^{m}\right)^{2}=\partial^{2} \operatorname{Re}(\zeta \hat{Z}) /\left(\partial \psi_{n}^{m}\right)^{2}
$$

This implies that

$$
T=\operatorname{Re}\left(\zeta \hat{Z}_{S}\right)+P \psi_{n}^{m}+Q \text { on } S,
$$

where $P, Q \in \mathcal{L}_{n}^{m}$. Note that the first term is a trivial conserved density; hence all that remains to be proven is that the linear term $P \psi_{n}^{m}$ can be incorporated into an $\mathbf{x}$-derivative term.

If $n \geqslant 1$, let $\hat{X}$ satisfy the equation

$$
\partial \hat{X} / \partial \psi_{n-1}^{m}=\left(1+\eta_{\mathbf{x}}\left(b_{n} / a_{n}\right)\right)^{-1} P \text { on } S .
$$

Then by (6.11),

$$
\begin{aligned}
\mathbf{D}_{\mathbf{x}} \hat{X} & =\frac{\partial \hat{X}}{\partial \psi_{n-1}^{m}} \cdot\left(\psi_{n}^{m}+\eta_{\mathbf{x}} \varphi_{n}^{m}\right)+Y^{\prime}=\frac{\partial \hat{X}}{\partial \psi_{n-1}^{m}} \cdot\left(1+\eta_{\mathbf{x}} \frac{b_{n}}{a_{n}}\right) \psi_{n}^{m}+Y^{\prime \prime} \\
& =P \psi_{n}^{m}+Y^{\prime \prime}
\end{aligned}
$$

where $Y^{\prime}, Y^{\prime \prime} \in \mathcal{L}_{n}^{m}$. Then

$$
T=\operatorname{Re}\left(\zeta \hat{Z}_{S}\right)+\mathbf{D}_{\mathbf{x}} \hat{X}+\bar{T}
$$

where $\bar{T} \in \mathcal{L}_{n}^{m}$. If, finally, $n=0$, then the only nontrivial $(m+1,0)$ order term in $\mathbf{D}_{\mathbf{t}} T$ is $P \psi_{0}^{m+1}$, and this can only arise from the analytic contribution in (7.5). An argument similar to the above shows that

$$
P \psi_{0}^{m+1}=\operatorname{Re}\left(\zeta Z^{*} \omega_{0}^{m+1}\right)+Y^{*} \quad \text { on } S ;
$$

hence

$$
P \psi_{0}^{m}=\operatorname{Re}\left(\zeta Z^{*} \omega_{0}^{m}\right)+Y^{* *} \text { on } S,
$$

where $Y^{*}, Y^{* *} \in \mathcal{E}_{0}^{m}$. In either case the induction is complete, in that

$$
T=\bar{T}+\operatorname{Re}(\zeta Z)+\mathbf{D}_{\mathbf{x}} W
$$

for $\bar{T} \in \mathcal{L}_{n}^{m}, Z$ analytic. This completes the proof of Lemma 9.1 .

10. A key lemma. In the following stages of the proof, we will often need to know when a function depending on surface derivatives of $\varphi, \eta$ is a trivial density. The following lemma answers a more general such question. 
LEMMA 10.1. Let $\kappa$ be a constant. A function $T \in C\left(\mathcal{V}_{S}^{\eta}\right)$ can be written as

$$
T=X+\kappa \eta_{\mathbf{x}} Y \text { on } S,
$$

where $Z=X+i Y$ is analytic in $\omega_{n}^{m}, z$, if and only if

$$
T=k(t) \varphi_{\mathbf{x}}+\left(\gamma+\kappa \eta_{\mathbf{x}} \delta\right) \text { on } S,
$$

where $k$ is a real-valued function of $t, k=0$ unless $\kappa=-1$, and $\gamma+i \delta$ is a function of $x, y, t$, analytic in $x+i y=z$.

Proof. Define the differential operator

$$
\nabla_{n}=b_{n}\left(\partial / \partial \varphi_{n}^{1}\right)+a_{n}\left(\partial / \partial \psi_{n}^{1}\right),
$$

where $a_{n}+i b_{n}=\zeta^{n}$. Taking spatial derivatives of (6.4), we find that

$$
\eta_{\mathrm{n}}=\left(\psi_{x t}+\beta\right)^{-1} \operatorname{Re}\left(\zeta^{n} \omega_{n}^{1}\right)+H_{n}, \quad n \geqslant 2,
$$

where $H_{n} \in \mathcal{L}_{n}^{1}$. From (4.14) we have

$$
\varphi_{\mathrm{n}}=\operatorname{Re}\left(\zeta^{n} \omega_{n}\right)-\eta_{\mathrm{n}} \psi_{x}+G_{n}, \quad n \geqslant 1,
$$

where $G_{n} \in \mathcal{L}_{n}^{1} \cap \mathcal{L}_{n}^{0}$. From these we conclude that

$$
\nabla_{n} \eta_{\mathrm{n}}=0=\nabla_{n} \varphi_{\mathrm{n}}, \quad n \geqslant 2 .
$$

Let $n \geqslant 2$ be the highest order surface derivative of $\eta$ and $\varphi$ occurring in $T$. Applying $\nabla_{n}$ to (10.1), we find (using the analyticity of $Z$ )

$$
0=\left(b_{n}+\kappa \eta_{\mathrm{x}} a_{n}\right) X_{\varphi_{n}^{1}}+\left(a_{n}-\kappa \eta_{\mathrm{x}} b_{n}\right) X_{\psi_{n}^{\prime}}
$$

hence

$$
Z=k_{n}(t) \zeta^{n}\left(1+i \kappa \eta_{\mathbf{x}}\right) \omega_{n}^{1}+Z_{0}
$$

where $k_{n}$ is real, $Z_{0} \in \mathcal{L}_{n}^{1}$. However, the dependence of $\eta_{\mathbf{x}}$ (hence $\zeta$ ) on $\omega_{x}, \omega_{x x}$ as given in (6.4) immediately implies that $Z$ cannot be analytic in these variables unless $k_{n}=0$.

Thus by induction $T$ only depends on first order derivatives of $\varphi$ and $\eta$. Let

$$
\nabla_{1}=\eta_{\mathbf{x}}\left(\partial / \partial \varphi_{x t}\right)+\partial / \partial \psi_{x t},
$$

which annihilates $T$; hence

$$
0=(1+\kappa) \eta_{\mathbf{x}} X_{\varphi_{x t}}+\left(1-\kappa \eta_{\mathbf{x}}^{2}\right) X_{\psi_{x t}} .
$$

For $\kappa \neq-1$, (6.4) shows that $X$ must be a function of the invariants $x, y, \varphi$ and $\left(\eta_{\mathbf{x}}^{-2}+1\right) \cdot\left(\varphi_{x t}+\alpha\right)^{2 \kappa /(\kappa+1)}$ of this first order partial differential equation, and it is easy to see that the only such analytic function $Z$ with this property is $Z=\gamma+i \delta$ satisfying the conclusion of the lemma. For $\kappa=-1,(10.4)$ shows that $Z=k(t) \omega_{x t}+$ $Z_{0}(x, y, t)$, which proves $(10.2)$ in this case.

In the course of the proof of the lemma, we have shown the following.

LEMMA 10.2. Let $\kappa$ be a constant. Suppose $T$ is a smooth function of one of the following sets of variables:

(a) $\mathscr{V}_{S}^{m}=\left\{x, t, \varphi_{n}, \psi_{n}, \varphi_{j}^{1}, \psi_{j}^{1}, \eta_{\mathbf{k}}: n \geqslant 0,0 \leqslant j<m, k \geqslant m\right\}$,

(b) $\overbrace{S}^{m}=\left\{x, t, \varphi_{j}, \psi_{j}, \varphi_{j}^{1}, \psi_{j}^{1}, \varphi_{\mathbf{k}}, \eta_{\mathbf{k}}: 0 \leqslant j<m, k \geqslant m\right\}$. 
and satisfies (10.1). Then $T$ is independent of

(a) $\eta_{\mathrm{n}}, n \geqslant m$,

(b) $\varphi_{\mathrm{n}}, \eta_{\mathrm{n}}, n \geqslant m$,

respectively.

The same proof also shows the following:

Lemma 10.3. A function $T \in C\left(\mathcal{V}_{S}^{\psi}\right)$ satisfies $T=X+\kappa \eta_{\mathbf{x}} Y$ on $S$ where $Z=X+$ $i Y$ is analytic and $\kappa$ is a constant if and only if $T \equiv k(t) \varphi_{t \mathbf{x}}+\left(X_{0}+\kappa \eta_{\mathbf{x}} Y_{0}\right)$, where $k=0$ if $\kappa \neq-1$, and $X_{0}+i Y_{0}$ is analytic in $z, \omega_{n}, n \geqslant 0$.

\section{Reduction to surface derivatives.}

LEMMA 11.1. Suppose $T \in C\left(\mathfrak{V}_{S}^{t}\right)$ is a conserved density. Then

$$
T=\bar{T}+k \varphi_{t \mathbf{x}} \psi_{t}+T_{0},
$$

where $\bar{T} \in C\left(\mathcal{V}_{S}^{\psi}\right), T_{0}$ is trivial, and $k(t)$ is a function of $t$ only.

Proof. Define the intermediate set of variables

$$
\mathcal{T}_{S}^{m}=\left\{x, t, \varphi_{n}, \psi_{n}, \varphi_{j}^{1}, \psi_{j}^{1}, \varphi_{\mathbf{k}}^{1}: n \geqslant 0,0 \leqslant j<m, k \geqslant m\right\} .
$$

Note that $\mathfrak{V}_{S}^{\infty}=\mathfrak{V}_{S}^{t}, \mathfrak{V}_{S}^{0}=\mathfrak{V}_{S}^{\psi}$. By the arguments of $\S 8, \mathcal{T}_{S}^{m}$ is equivalent to the set

$$
\overline{\mathcal{V}}_{S}^{m}=\left\{x, t, \varphi_{n}, \psi_{n}, \varphi_{\mathrm{n}}^{1}, \psi_{j}^{1}: n \geqslant 0,0 \leqslant j<m\right\}
$$

Suppose by induction we have shown that $T$ is equivalent to a density (which we also call $T$ ) in $C\left(\mathscr{V}_{S}^{n+1}\right), n \geqslant 1$. Letting $R_{j}$ denote various functions in $\mathcal{L}_{0}^{2}$, integration by parts, (4.18) and (6.9) show that

$$
\begin{aligned}
\mathbf{D}_{\mathbf{t}} T & =\sum_{j=0}^{n}\left(T_{\varphi_{j}^{\prime}} \varphi_{j}^{2}+T_{\psi_{j}^{\prime}} \psi_{j}^{2}\right)+R_{1}=\sum_{j=0}^{n}\left(-d_{j} T_{\varphi_{j}^{\prime}}+c_{j} T_{\psi_{j}^{\prime}}\right) \psi_{\mathrm{j}}^{2}+R_{2} \\
& =A \psi_{t t}+R_{3}+\mathbf{D}_{\mathbf{x}} X,
\end{aligned}
$$

where

$$
A=\sum_{j=0}^{n}\left(-\mathbf{D}_{\mathbf{x}}\right)^{j}\left(-d_{j} T_{\varphi_{j}^{\prime}}+c_{j} T_{\psi_{j}^{\prime}}\right) .
$$

Abbreviate $u=\varphi_{n}^{1}, v=\psi_{n}^{1}$. Then

$$
\begin{aligned}
A & =(-1)^{n}\left\{\left(c_{n} T_{v v}-d_{n} T_{u v}\right) \mathbf{D}_{\mathbf{x}}^{n} \psi_{n}^{1}+\left(c_{n} T_{u v}-d_{n} T_{u u}\right) \mathbf{D}_{\mathbf{x}}^{n} \varphi_{n}^{1}\right\}+B_{1} \\
& =(-1)^{n}\left\{P \varphi_{2 n}^{1}-Q \psi_{2 n}^{1}\right\}+B_{2},
\end{aligned}
$$

for $B_{j} \in \mathcal{L}_{2 n}^{1}$, and

$$
P=T_{u v}+b_{n} c_{n} \Delta T, \quad Q=T_{u u}-a_{n} c_{n} \Delta T,
$$

with $\Delta=\partial_{u}^{2}+\partial_{v}^{2}$.

Only the analytic terms in (7.5) can contribute to the term $A \psi_{t t}$ in $\mathbf{D}_{\mathbf{t}} T$; hence $Z=(-1)^{n} \hat{Z} \omega_{2 n}^{1} \omega_{t t}+Z_{0}$, where $Z_{0} \in \mathcal{L}_{2 n}^{1}$. Also

$$
P=-\hat{Y}-\eta_{\mathbf{x}} \hat{X}, \quad Q=\hat{X}-\eta_{\mathbf{x}} \hat{Y},
$$


where $\hat{Z}=\hat{X}+i \hat{Y}$. Since $\hat{Z}$ is analytic, $P$ and $Q$ satisfy the Cauchy-Riemann equations in $u, v$, so from (11.3),

$$
\Delta\left(b_{n} T_{u}+a_{n} T_{v}\right)=0 .
$$

We infer that $T=T^{1}+T^{2}$, where $T^{1} \in C\left(\overline{\mathscr{V}}_{S}^{n}\right)$ and $T^{2}$ is harmonic in $u, v$, satisfying (11.4) with $P=T_{u v}^{2}, Q=T_{u u}^{2}$. Therefore if $Z^{2}$ is an analytic function such that $\partial^{2} Z^{2} /\left(\partial \omega_{n}^{1}\right)^{2}=-\hat{Z}$, then $T^{2}$ and $\operatorname{Re}\left(\zeta Z^{2}\right)$ agree up to linear terms in $\varphi_{n}^{1}$ and $\psi_{n}^{1}$. Hence

$$
T^{2}=\operatorname{Re}\left(\zeta Z^{2}\right)+A^{2} \varphi_{\mathbf{n}}^{1}+B^{2} \psi_{\mathbf{n}}^{1}+C^{2},
$$

where $A^{2}, B^{2}, C^{2} \in C\left(\overline{\mathscr{V}}_{S}^{n}\right)$, with $A^{2}, B^{2}$ independent of $\varphi_{\mathrm{n}}^{1}$. Note further that from Lemma $10.2(\mathrm{a}), Z^{2}$ is independent of $\varphi_{\mathrm{m}}^{1}$ for $m \geqslant n$. Finally, to eliminate the linear $\psi_{\mathrm{n}}^{1}$ term, represent $B^{2}$ as an element of $C\left(\overline{\mathscr{V}}_{S}^{n}\right)$ and choose $R^{2} \in C\left(\overline{\mathscr{V}}_{S}^{n}\right)$ satisfying $\partial R^{2} / \partial \psi_{n-1}^{1}=B^{2}$. Then

$$
T=T^{1}+T^{2}=\left(T^{1}+A^{2} \varphi_{\mathbf{n}}^{1}+\hat{C}^{2}\right)+\left(\operatorname{Re}\left(\zeta Z^{2}\right)+\mathbf{D}_{\mathbf{x}} R^{2}\right) .
$$

The first group of terms is in $C\left(\overline{\mathscr{V}}_{S}^{n}\right)$, the second group is trivial. Thus by induction we can assume $T \in C\left(\overline{\mathcal{V}}_{S}^{2}\right)$.

The above argument must be slightly modified when $n=1$. Replacing (11.2), we have

$$
A=-P \varphi_{t x x}+Q \psi_{t x x}+B_{2}
$$

where $B_{2} \in \mathcal{L}_{2}^{1}$, and

$$
P=A_{u}^{1}+\eta_{\mathbf{x}} A_{v}^{1}, \quad Q=-A_{v}^{1}+\eta_{\mathbf{x}} A_{u}^{1},
$$

where $u=\varphi_{x t}, v=\psi_{x t}, A^{1}=-d_{1} T_{u}+c_{1} T_{v}$. The analogue of (11.5) then holds since, from (11.4),

$$
\hat{X}=-A_{u}^{1}, \quad \hat{Y}=-A_{v}^{1} .
$$

From this it is easily checked that the rest of the proof goes through.

Finally, we must deal with the case $T \in C\left(\mathcal{V}_{S}^{1}\right)$. The only term involving $\psi_{t t}$ in $\mathbf{D}_{\mathbf{t}} T$ is $T_{\psi_{t}} \psi_{t t}$, hence $T_{\psi_{t}}=\operatorname{Re}(\zeta \hat{Z})$ for some analytic function $\hat{Z}$. By a slight modification of Lemma 10.3,

$$
T_{\psi_{t}}=k(t) \varphi_{t \mathbf{x}}+\operatorname{Re}\left(\zeta \hat{Z}^{\prime}\right)
$$

where $\hat{Z}^{\prime}$ is analytic in $z, \omega_{n}, n \geqslant 0$, and $\omega_{t}$. Let $\partial_{\omega_{t}} Z^{*}=-\hat{Z}^{\prime}$. Then

$$
T=k(t) \varphi_{t \mathbf{x}} \psi_{t}+\bar{T}+\operatorname{Re}\left(\zeta Z^{*}\right)
$$

where $\bar{T} \in C\left(\overline{\mathscr{V}}_{S}^{0}\right)$, which completes the proof of the lemma.

\section{Elimination of the stream function.}

LEMMA 12.1. Suppose the density $T+k(t) \varphi_{t \mathbf{x}} \psi_{t}$ is conserved, where $T \in C\left(\mathfrak{V}_{S}^{\psi}\right)$. Then $k=0$, and $T=\bar{T}+\bar{k} \varphi_{\mathbf{x}} \psi+T_{0}$, where $\bar{T} \in C\left(\mathscr{V}_{S}^{\varphi}\right), T_{0}$ is trivial, and $\bar{k}$ depends only on $t$. 
Proof. The proof is very similar to that of the preceding lemma, so we omit most of the details. Introduce the sets of variables

$$
\overline{\mathcal{V}}_{S}^{m}=\left\{x, t, \varphi_{j}, \psi_{j}, \varphi_{\mathbf{k}}, \eta_{\mathbf{n}}: n \geqslant 0,0 \leqslant j<m, k \geqslant m\right\},
$$

or, equivalently,

$$
\hat{\vartheta}_{S}^{m}=\left\{x, t, \varphi_{\mathrm{n}}, \eta_{\mathrm{n}}, \psi_{\mathbf{j}}: n \geqslant 0,0 \leqslant j<m\right\} .
$$

Assume by induction that $T \in C\left(\overline{\mathcal{V}}_{S}^{n+1}\right)$ for $n \geqslant 2$. Analysis of the highest order terms in $D_{t} T$ leads to equations of the same form as (11.4), (11.5), but with $u=\varphi_{n}$, $v=\psi_{n}$. The rest of the proof proceeds word for word as before, only Lemma 10.2(b) must now be invoked. Thus we are reduced to the case $T \in C\left(\overline{\mathscr{V}}_{S}^{2}\right)$.

Analysis of the first order terms is slightly complicated by the $k(t) \varphi_{t \mathrm{x}} \psi_{t}$ term. It is, however, reasonably easy to prove that $k=0$ before analyzing the other terms. Note that if $\mathbf{D}_{\mathbf{t}} T$ is expressed in terms of the variables in $\overline{\mathcal{V}}_{S}^{t}$, then quadratic terms in $\psi_{\mathbf{n}}^{1}$ can only arise from the $k \varphi_{t \mathbf{x}} \psi_{t}$ term. We have

$$
\mathbf{D}_{\mathbf{t}}\left(k \varphi_{t \mathbf{x}} \psi_{t}\right)=k\left(\varphi_{t \mathbf{x} \mathbf{x}} \psi_{t}+\varphi_{t \mathbf{x}} \psi_{t \mathbf{t}}\right)+k_{t} \varphi_{t \mathbf{x}} \psi_{t},
$$

which must be expressed in terms of surface derivatives of $\eta$ and $\psi_{t}$. (For the derivatives of $\varphi_{t}$, recall the definition (6.1) of $\eta$.) From (6.6) and (4.13) we have

$$
\varphi_{t t}=-2\left(1+\eta_{\mathbf{x}}^{2}\right)^{-1}\left(\varphi_{t \mathbf{x}} \varphi_{\mathbf{x}}+\psi_{t \mathbf{x}} \psi_{\mathbf{x}}\right)+G=-2 \xi \psi_{t \mathbf{x}}+H
$$

where $\xi=\left(1+\eta_{\mathbf{x}}^{2}\right)^{-1} \psi_{\mathbf{x}}, G \in \mathcal{L}_{0}^{1}$, and $H$ does not depend on $\psi_{t}$ or its surface derivatives. Then from (3.6), (4.20),

$$
\varphi_{t \mathbf{t x}}=D_{\mathbf{x}}\left(\varphi_{t t}+\psi_{\mathbf{x}} \psi_{t x}\right)=-D_{\mathbf{x}}\left(\xi \psi_{t \mathbf{x}}\right)+\bar{H}
$$

$\bar{H}$ of the same form as $H$. Furthermore,

$$
\psi_{t \mathbf{t}}=\psi_{t t}-\xi\left(\varphi_{t \mathbf{x}}+\eta_{\mathbf{x}} \psi_{t \mathbf{x}}\right)
$$

hence only $\varphi_{t \mathrm{x}} \psi_{t t}$ in the second term of (12.1) makes a contribution to the quadratic $\psi_{t}$ terms. Since

$$
\operatorname{Re}\left(-i \zeta \omega_{t t} \omega_{t x}\right)=\varphi_{t t} \psi_{t \mathbf{x}}+\psi_{t t} \varphi_{t \mathbf{x}}
$$

we find that

$$
\mathbf{D}_{\mathbf{t}}\left(k \varphi_{t \mathbf{x}} \psi_{t}\right)=k\left[\xi\left(2 \psi_{t \mathbf{x}}^{2}-\psi_{t \mathbf{x}} \psi_{t}\right)-\xi_{\mathbf{x}} \psi_{t \mathbf{x}} \psi_{t}\right]+L
$$

where $L$ is a sum of terms linear in $\psi_{t}$ and its $\mathbf{x}$-derivatives, and a trivial density.

On the other hand, the uniqueness results in Proposition 5.4 imply that the only nonduplicative quadratic terms in $Z$ and $W$ which can contribute to (7.5) are

$$
Z=Z_{1}\left(\omega_{t}\right)^{2}+Z_{2}\left(\omega_{t x}\right)^{2}+Z_{0}, \quad W=W_{1}\left(\psi_{t}\right)^{2}+W_{2} \psi_{t} \psi_{t \mathbf{x}}+W_{0},
$$

where $Z_{0}, W_{0}$ are linear in $\psi_{t}$ and its derivatives. Note that from (4.13),

$$
\operatorname{Re}\left(\zeta Z_{2}\left(\omega_{t x}\right)^{2}\right)=\operatorname{Re}\left(\zeta^{-1} Z_{2}\left(\omega_{t \mathbf{x}}\right)^{2}\right)=-\operatorname{Re}\left(\zeta^{-1} Z_{2}\right)\left(\psi_{t \mathbf{x}}\right)^{2}+\cdots ;
$$

hence the quadratic terms in $\operatorname{Re}(\zeta Z)+\mathbf{D}_{\mathbf{x}} W$ are

$$
\begin{aligned}
\left(\mathbf{D}_{\mathbf{x}} W_{1}\right. & \left.-\operatorname{Re}\left(\zeta Z_{1}\right)\right)\left(\psi_{t}\right)^{2}+\left(2 W_{1}+D_{\mathbf{x}} W_{2}\right) \psi_{t \mathbf{x}} \psi_{t} \\
& +\left(W_{2}-\operatorname{Re}\left(\zeta^{-1} Z_{2}\right)\right)\left(\psi_{t \mathbf{x}}\right)^{2}+W_{2} \psi_{t \mathbf{x} \mathbf{x}} \psi_{t} .
\end{aligned}
$$


Equating this to (12.3) we find, in particular, that $W_{2}=-k \xi$, and, hence, $\operatorname{Re}\left(\zeta^{-1} Z_{2}\right)$ $=-3 k \xi$. This is equivalent to

$$
X_{2}+\eta_{\mathbf{x}} Y_{2}=-3 k \psi_{\mathbf{x}}=-3 k\left(\psi_{x}+\eta_{\mathbf{x}} \varphi_{x}\right)
$$

where $Z_{2}=X_{2}+i Y_{2}$. But this would mean that $Z_{2}=-3 k\left(\psi_{x}+i \varphi_{x}\right)$, which is not analytic. Therefore $k=0$.

The remaining first order terms in $T$ are treated much the same way as the first order terms were in the proof of Lemma 11.1. For brevity we omit the details.

\section{Higher order surface derivatives.}

Lemma 13.1. Suppose $T+k(t) \varphi_{\mathrm{x}} \psi$, where $T \in C\left(\mathcal{V}_{S}^{\varphi}\right)$, is conserved. Then

$$
T=\bar{T}+\left(\gamma+\eta_{\mathbf{x}} \delta\right) \varphi \text { on } S,
$$

where $\bar{T} \in C\left(\mathcal{V}_{S}^{\eta}\right)$ and $\gamma+i \delta$ is a function of $x, y, t$, analytic in $x+i y$.

Proof. Let $\mathbf{E}_{\varphi}=\Sigma\left(-\mathbf{D}_{\mathbf{x}}\right)^{n} \partial_{\varphi_{\mathbf{n}}}$ denote the variational derivative with respect to $\varphi$, and similarly for $\mathbf{E}_{\eta}$. Let

$$
\tilde{\mathbf{D}}_{\mathbf{t}} T=\mathbf{E}_{\varphi}(T) \varphi_{\mathbf{t}}+\mathbf{E}_{\eta}(T) \eta_{\mathbf{t}}+T_{t}
$$

so that $\mathbf{D}_{\mathbf{t}} T=\tilde{\mathbf{D}}_{\mathbf{t}} T+\mathbf{D}_{\mathbf{x}} X$ for some $X$.

Consider the operator

$$
\partial_{\psi_{x}}=\left(1+\eta_{\mathbf{x}}^{2}\right)^{-1}\left(\partial_{\psi_{x}}+\eta_{\mathbf{x}} \partial_{\varphi_{x}}\right)
$$

From (4.20) we have

$$
\partial_{\psi_{\mathbf{x}}}\left(\eta_{\mathbf{t}}\right)=-1, \quad \partial_{\psi_{\mathbf{x}}}\left(\varphi_{\mathbf{t}}\right)=\psi_{x},
$$

and

$$
\partial_{\psi_{\mathbf{x}}}^{2}\left(\eta_{\mathbf{t}}\right)=0, \quad \partial_{\psi_{\mathbf{x}}}^{2}\left(\varphi_{\mathbf{t}}\right)=\left(1+\eta_{\mathbf{x}}^{2}\right)^{-1}
$$

Therefore

$$
\partial_{\psi_{\mathbf{x}}}^{2} \tilde{\mathbf{D}}_{\mathbf{t}}\left(T+k \varphi_{\mathbf{x}} \psi\right)=\left(1+\eta_{\mathbf{x}}^{2}\right)^{-1} \mathbf{E}_{\varphi}(T) .
$$

On the other hand, since (7.5) reads

$$
\tilde{\mathbf{D}}_{\mathbf{t}}\left(T+k \varphi_{\mathbf{x}} \psi\right)=\operatorname{Re}(\zeta Z)+\mathbf{D}_{\mathbf{x}} \tilde{W}, \quad \tilde{W}=W-X,
$$

for some analytic $Z$, by the uniqueness results of Proposition $5.4, W$ must be independent of $\psi_{x}$ since $\psi_{x x}$ does not appear on the left-hand side of (13.6), or linearly in $\operatorname{Re}(\zeta Z)$. Therefore

$$
\partial_{\psi_{\mathbf{x}}}^{2}\left(D_{\mathbf{x}} \tilde{W}\right)=\partial_{\psi_{\mathbf{x}}} \partial_{\psi} \tilde{W}=0
$$

Also, an easy computation shows:

$$
\begin{gathered}
\partial_{\psi_{\mathbf{x}}}(\operatorname{Re} \zeta Z)=-Y_{\varphi_{x}}, \\
\partial_{\psi_{\mathbf{x}}}^{2}(\operatorname{Re} \zeta Z)=-\left(1+\eta_{\mathbf{x}}^{2}\right)^{-1}\left(X_{\varphi_{x} \varphi_{x}}+\eta_{\mathbf{x}} Y_{\varphi_{x} \varphi_{x}}\right) .
\end{gathered}
$$

Therefore, applying $\partial_{\psi_{x}}^{2}$ to (13.6) and using (13.5), (13.7), (13.9) we find that

$$
\mathbf{E}_{\varphi}(T)=-\left(X_{\varphi_{x} \varphi_{x}}+\eta_{\mathbf{x}} Y_{\varphi_{x} \varphi_{x}}\right) .
$$


Now from Lemma 10.1, with $\kappa=+1$, we conclude that

$$
\mathbf{E}_{\varphi}(T)=\gamma+\eta_{\mathbf{x}} \delta \quad \text { on } S
$$

where $\gamma+i \delta$ is analytic in $x+i y$. The well-known property of the variational derivative that

$$
\text { kernel } \mathbf{E}_{\varphi}=C\left(\mathcal{V}_{S}^{\eta}\right)+\text { image } \mathbf{D}_{\mathbf{x}},
$$

see for instance [19], completes the proof of Lemma 13.1.

LEMMA 13.2. If the expression

$$
T=\bar{T}+\left(\gamma+\eta_{\mathbf{x}} \delta\right) \varphi+k(t)\left(\varphi_{\mathbf{x}} \psi+g \eta^{2}\right),
$$

where $\bar{T} \in C\left(\mathcal{V}_{S}^{\eta}\right)$, and $\gamma, \delta$ depend only on $x, y, t$, is a conserved density, then $\bar{T}$ is a function of $x, y, t$ only, harmonic in $x, y$, and

$$
\gamma+i \delta=\left(l+\frac{1}{2} i k_{t}\right)(x+i y)+m+i n,
$$

where $l, m, n$ are real-valued functions of $t$.

Proof. Note first that since $\frac{1}{2}\left(\varphi_{x} \psi+g \eta^{2}\right)$ represents the energy density $T_{2}$, its time derivative is already of the form (7.5) [4]. The only other term in $\tilde{\mathbf{D}}_{\mathbf{t}} T$ involving $\psi$ is $k_{t} \varphi_{\mathbf{x}} \psi$; hence

$$
k_{t} \varphi_{\mathbf{x}}=\operatorname{Re}\left(\zeta Z_{\psi}\right)+\mathbf{D}_{\mathbf{x}} \tilde{W}_{\psi} .
$$

By the uniqueness results on $Z$ and $W$, we must have

$$
\tilde{W}=\left(k_{t} \varphi-\varepsilon\right) \psi+W^{0} \text { on } S, \quad Z=2 i \omega \partial_{z} \varepsilon+Z^{0},
$$

where $\varepsilon(x, y, t)$ is harmonic, $W^{0}$ is independent of $\psi$ and $Z^{0}=X^{0}+i Y^{0}$ is independent of $\omega$.

Next apply $\partial_{\psi_{\mathrm{x}}}$ to (13.6) and use (13.3), (13.8) to find that

$$
\gamma \psi_{x}+\delta \varphi_{x}-\left(\delta_{x}+\gamma_{y}\right) \varphi-\mathbf{E}_{\eta}(\bar{T})=-Y_{\varphi_{x}}^{0}+k_{t} \varphi-\varepsilon \text { on } S .
$$

Let $Z^{0}=-\frac{1}{2}(\gamma+i \delta)\left(\omega_{x}\right)^{2}+\hat{Z}^{0}$, so

$$
\varepsilon+\hat{Y}_{\varphi_{x}}^{0}=\mathbf{E}_{\eta}(\bar{T})+\left(k_{t}+2 \gamma_{y}\right) \varphi \text { on } S .
$$

From Lemma 10.1 with $\kappa=0$, and the uniqueness properties,

$$
\hat{Y}_{\varphi_{x}}^{0}=0, \quad \mathbf{E}_{\eta}(\bar{T})=\varepsilon, \quad \text { and } \quad k_{t}=-2 \gamma_{y} .
$$

This completes the proof of the lemma.

14. First order conservation laws. Recapping $\$ \S 9-13$, it has been shown that the only nontrivial conserved densities for the water wave problem are of the form

$$
T=\left(\gamma+\eta_{\mathbf{x}} \delta\right) \varphi+\bar{T}+k\left(\varphi_{\mathbf{x}} \psi+g \eta^{2}\right) \text { on } S,
$$

where $\gamma, \delta, \bar{T}$ depend on $x, y, t, \gamma+i \delta$ is of the form (13.11), and $\varepsilon=\bar{T}_{\eta}$ is harmonic in $x, y$. It remains to prove that all densities of this form must be equivalent to a linear combination of the eight known conserved densities.

A closer look at the proofs in the previous sections reveals that

$$
Z=-\frac{1}{2}(\gamma+i \delta)\left(\omega_{x}\right)^{2}+2 i \omega \varepsilon_{z}, \quad W=\left(k_{t} \varphi-\varepsilon\right) \psi+W^{0},
$$


where $W^{0}$ depends on $x, t, \eta, \varphi$. Substituting (14.1), (14.2) into (7.5) and equating the coefficients of the various derivatives of $\varphi, \eta$, we are led to the final conditions

$$
\begin{array}{lll}
\delta_{t}=\varepsilon_{x}, \quad \gamma_{t}=-\varepsilon_{y}, \quad W_{\varphi}^{0}=0, & W_{\eta}^{0}=-g \delta \eta, \\
& W_{x}^{0}=\bar{T}_{t}-g \eta \gamma+k_{t} g \eta^{2} \quad \text { on } S .
\end{array}
$$

The general solution of (14.3) is given by (14.2), with

$$
\begin{gathered}
\bar{T}=n_{t} x \eta-\frac{1}{2} m_{t} \eta^{2}+r \eta+\bar{T}_{0}, \\
W^{0}=-\frac{1}{2} g k_{t} x \eta^{2}-\frac{1}{3} g l \eta^{3}+\frac{1}{2} n \eta^{2}+\left(r_{t}-g m\right) x \eta,
\end{gathered}
$$

where $\bar{T}_{0}$ is trivial, and

$$
\begin{gathered}
k=2 c_{7} t+\frac{1}{2} c_{2}, \quad l=c_{8}, \quad m=\frac{7}{2} g c_{7} t^{2}-c_{6} t+c_{4}, \\
n=\frac{1}{2} g c_{8} t^{2}+c_{5} t+c_{1}, \quad r=\frac{7}{6} g^{2} c_{7} t^{3}-\frac{1}{2} g c_{6} t^{2}+g c_{4} t+c_{3},
\end{gathered}
$$

where $c_{1}, \ldots, c_{8}$ are arbitrary constants. But then

$$
T=\sum_{i=1}^{8} c_{i} T_{i},
$$

where $T_{1}, \ldots, T_{8}$ are the densities in (1.5). This completes the proof of the main theorem; only these eight densities are conserved.

\section{REFERENCES}

1. C. J. Amick and J. F. Toland, On solitary water waves of finite amplitude, Arch. Rational Mech. Anal. 76 (1981), 9-95.

2. T. B. Benjamin, J. L. Bona and J. J. Mahony, Model equations for long waves in nonlinear dispersive systems, Philos. Trans. Roy. Soc. London Ser. A 272 (1972), 47-78.

3. T. B. Benjamin and J. J. Mahony, On an invariant property of water waves, J. Fluid. Mech. 49 (1971), 385-389.

4. T. B. Benjamin and P. J. Olver, Hamiltonian structure, symmetries and conservation laws of water waves, J. Fluid. Mech. 125 (1982).

5. D. J. Benney, Some properties of long nonlinear waves, Stud. Appl. Math. 52 (1973), 45-50.

6. J. L. Bona, W. G. Pritchard and L. R. Scott, Solitary wave interaction, Phys. Fluids 23 (1980), 438-441.

7. L. Comtet, Une formule explicite pour les puissances successives de l'opérateur de dérivation de Lie, C. R. Acad. Sci. Paris 276A (1973), 165-168.

8. K. O. Friedrichs and D. H. Hyers, The existence of solitary waves, Comm. Pure Appl. Math. 7 (1954), 517-550.

9. C. S. Gardner, Korteweg-deVries equation and generalizations. IV: The Korteweg-deVries equation as a Hamiltonian system, J. Math. Phys. 12 (1971), 1548-1551.

10. D. J. Korteweg and G. deVries, On the change of form of long waves advancing in a rectangular canal and on a new type of long stationary waves, Philos. Mag. 39 (1895), 422-443.

11. G. L. Lamb, Elements of soliton theory, Wiley, New York, 1980.

12. H. Lamb, Hydrodynamics, Cambridge Univ. Press, London, 1932.

13. M. S. Longuet-Higgins, $A$ theory of the origin of microseisms, Philos. Trans. Roy. Soc. London Ser. A 243 (1950), 1-35.

14. __ Spin and angular momentum in gravity waves, J. Fluid Mech. 97 (1980), 1-25.

15. Yu. I. Manin, Algebraic aspects of nonlinear differential equations, J. Soviet Math. 11 (1979), 1-122.

16. H. P. McKean and E. Trubowitz, Hill's operator and hyperelliptic function theory in the presence of infinitely many branch points, Comm. Pure Appl. Math. 29 (1976), 143-226.

17. V. I. Nalimov, $A$ priori estimates of elliptic equations in the class of analytic functions and their applications to the Cauchy-Poisson problem, Soviet Math. Dokl. 189 (1969), 1350-1354. 
18. P. J. Olver, Euler operators and conservation laws of the BBM equation, Math. Proc. Cambridge Philos. Soc. 85 (1979), 143-160.

19. __ Applications of Lie groups to differential equations, Oxford Univ. Lecture Notes, 1980.

20. A nonlinear Hamiltonian structure for the Euler equation, J. Math. Anal. Appl. 89 (1982), 233-250.

21. J. Reeder and M. Shinbrot, The initial value problem for surface waves under gravity. II: The simplest 3-dimensional case, Indiana Univ. Math. J. 25 (1976), 1049-1071.

22. __ The initial value problem for surface waves under gravity. III: Uniformly analytic domains, J. Math. Anal. Appl. 67 (1979), 340-391.

23. J. Riordan, Combinatorial identities, Wiley, New York, 1968.

24. M. Shinbrot, The initial value problem for surface waves under gravity. I: The simplest case, Indiana Univ. Math. J. 25 (1976), 281-300.

25. J. J. Stoker, Water waves, Wiley, New York, 1957.

26. W. A. Strauss, Nonlinear invariant wave equations, Invariant Wave Equations, Lecture Notes in Physics, vol. 73, Springer-Verlag, New York, 1978.

27. G. B. Whitham, Linear and nonlinear waves, Wiley, New York, 1974.

28. E. T. Whittaker, $A$ treatise on the analytical dynamics of particles and rigid bodies, Cambridge Univ. Press, London, 1965.

29. H. Yosihara, Gravity waves on the free surface of an incompressible perfect fluid of finite depth, Publ. Res. Inst. Math. Sci. Kyoto Univ. 18 (1982), 49-96.

30. V. E. Zakharov, Stability of periodic waves of finite amplitude on the surface of a deep fluid, J. Appl. Math. Tech. Phys. 9 (1968), 86-94.

School of Mathematics, University of Minnesota, Minneapolis, Minnesota 55455 